\title{
Triple-Quark Elastic Scatterings and Thermalization
}

\author{
Xiao-Ming $\mathrm{Xu}^{\mathrm{a}, \mathrm{b}}$, Peng $\mathrm{Ru}^{\mathrm{c}}$, H. J. Weber ${ }^{\mathrm{d}}$ \\ aDepartment of Physics, Shanghai University, Baoshan, Shanghai 200444, China \\ ${ }^{\mathrm{b}}$ Nuclear Physics Division, Shanghai Institute of Applied Physics \\ Chinese Academy of Sciences, P.O.Box 800204, Shanghai 201800, China \\ ${ }^{\mathrm{c}}$ Department of Physics, Wuhan University of Science and Technology, Wuhan 430081, China \\ ${ }^{\mathrm{d} D e p a r t m e n t}$ of Physics, University of Virginia, Charlottesville, VA 22904, U.S.A.
}

\begin{abstract}
Triple-quark elastic scattering amplitudes from perturbative QCD are first calculated and then used in a transport equation to study the thermalization of quark matter. By examining momentum isotropy to which the transport equation leads, we can determine thermalization time and offer an initial thermal quark distribution function. With an anisotropic initial quark distribution, which is relevant to quark matter initially created in a central $\mathrm{Au}-\mathrm{Au}$ collision at $\sqrt{s_{N N}}=200 \mathrm{GeV}$, the transport equation gives a time of the order of $1.8 \mathrm{fm} / c$ for quark matter itself to thermalize by the triple-quark scatterings.
\end{abstract}

PACS codes: 24.85.+p; 12.38.Mh; 25.75.Dw; 25.75.Gz

Keywords: Triple-quark scattering; transport equation; thermalization 


\section{Introduction}

Hydrodynamic calculations [1-7] that explain the elliptic flow coefficient $\mathrm{v}_{2}[8,9]$ at $p_{\perp}<2 \mathrm{GeV} / c$, lead to the conclusion that quark-gluon matter created in $\mathrm{Au}-\mathrm{Au}$ collisions at the Relativistic Heavy Ion Collider (RHIC) thermalizes rapidly. The usual parton scatterings give a long thermalization time of quark-gluon matter [10]. However, triple-gluon elastic scatterings in gluon matter lead to a short thermalization time of the order of $0.45 \mathrm{fm} / c$ [11]. Such triple-gluon elastic scatterings can occur in view of the high gluon number density that has been achieved in Au-Au collisions [12-14]. However, since quark-gluon matter includes quarks and measured quantities rely on the presence of quarks, it is important to investigate the thermalization of quark matter based on initial anisotropic quark distributions. At present, the thermalization mechanism of quark matter is unknown. In this letter, we study the thermalization based on two- and threequark elastic scattering processes. Until now, the squared amplitudes for the triple-quark scatterings have not been studied and must be calculated in perturbative QCD. Therefore, this work comprises two new tasks: one is to derive the squared amplitudes and the other is to study the thermalization. In order to keep the physics relating to the triple-quark elastic scatterings transparent, and due to the complications of calculating the squared amplitudes and numerically solving a transport equation, we exclude here the quark scatterings with gluons and/or antiquarks.

\section{Quark-quark and triple-quark scatterings}

The evolution of quark matter relies on the scattering of two quarks with identical or different flavors. Expressed in terms of the Mandelstam variables $(s, t, u)$, the spinand color-averaged squared amplitudes of order $\alpha_{\mathrm{s}}^{2}$ in perturbative QCD $[15,16]$ are

$$
\left|\mathcal{M}_{\mathrm{uu} \rightarrow \mathrm{uu}}\right|^{2}=\left|\mathcal{M}_{\mathrm{dd} \rightarrow \mathrm{dd}}\right|^{2}=\mathrm{g}_{\mathrm{s}}^{4}\left[\frac{4}{9}\left(\frac{s^{2}+u^{2}}{t^{2}}+\frac{s^{2}+t^{2}}{u^{2}}\right)-\frac{8}{27} \frac{s^{2}}{u t}\right]
$$

for the scattering of two up (down) quarks and

$$
\left|\mathcal{M}_{\mathrm{ud} \rightarrow \mathrm{ud}}\right|^{2}=\mathrm{g}_{\mathrm{s}}^{4} \frac{4}{9} \frac{s^{2}+u^{2}}{t^{2}}
$$

for the scattering of up and down quarks. Here, $\mathrm{g}_{\mathrm{s}}$ is the quark-gluon coupling constant and $\mathrm{g}_{\mathrm{s}}^{2}=4 \pi \alpha_{\mathrm{s}}$.

The diagrams for elastic scattering processes of three quarks at order $\alpha_{\mathrm{s}}^{4}$ are drawn in Figs. 1 and 2. The three-quark scattering processes in Fig. 1 and Fig. 2 are separated into two distinct classes of diagrams:

(1) $\mathrm{A}_{-}, \mathrm{A}_{-45}, \mathrm{~A}_{-56}, \mathrm{~A}_{-64}, \mathrm{~A}_{-4(56)}, \mathrm{A}_{-(45) 6}$ of Fig. 1

involving two successive gluon exchanges and,

(2) $\mathrm{A}_{*}, \mathrm{~A}_{* 45}, \mathrm{~A}_{* 56}, \mathrm{~A}_{* 64}, \mathrm{~A}_{* 4(56)}, \mathrm{A}_{*(45) 6}$ in Fig. 2,

involving a triple gluon coupling.

When two final quarks have the same flavor, an exchange of the two quarks generates a different diagram from those diagrams $A_{-}$in Fig. 1 or $A_{*}$ in Fig. 2. When three quarks have the same flavor, exchanges of the three quarks lead to the diagrams $A_{-4(56)}$ and $\mathrm{A}_{-(45) 6}$ of Fig. 1 and $\mathrm{A}_{* 4(56)}$ and $\mathrm{A}_{*(45) 6}$ of Fig. 2. 
A quark four-momentum is labeled as $p_{\mathrm{i}}=\left(E_{\mathrm{i}}, \vec{p}_{\mathrm{i}}\right)$ in the process $\mathrm{q}\left(p_{1}\right)+\mathrm{q}\left(p_{2}\right)+$ $\mathrm{q}\left(p_{3}\right) \rightarrow \mathrm{q}\left(p_{4}\right)+\mathrm{q}\left(p_{5}\right)+\mathrm{q}\left(p_{6}\right)$. The six momentum variables are assigned to quarks in six orderings. For instance, even though the momenta $p_{1}, p_{2}$ and $p_{3}$ may be assigned to the initial quarks from left to right of a diagram, while $p_{4}, p_{5}$ and $p_{6}$ are assigned to the final quarks from left to right, the initial quarks may also take the order of $\left(p_{1}, p_{3}, p_{2}\right)$ and correspondingly the final quarks have momenta in the order of $\left(p_{4}, p_{6}, p_{5}\right)$. If these two orderings are denoted as

$$
\left(\begin{array}{lll}
p_{4} & p_{5} & p_{6} \\
p_{1} & p_{2} & p_{3}
\end{array}\right),\left(\begin{array}{lll}
p_{4} & p_{6} & p_{5} \\
p_{1} & p_{3} & p_{2}
\end{array}\right)
$$

the other four orderings are written as

$$
\left(\begin{array}{lll}
p_{5} & p_{4} & p_{6} \\
p_{2} & p_{1} & p_{3}
\end{array}\right),\left(\begin{array}{lll}
p_{5} & p_{6} & p_{4} \\
p_{2} & p_{3} & p_{1}
\end{array}\right),\left(\begin{array}{lll}
p_{6} & p_{4} & p_{5} \\
p_{3} & p_{1} & p_{2}
\end{array}\right),\left(\begin{array}{lll}
p_{6} & p_{5} & p_{4} \\
p_{3} & p_{2} & p_{1}
\end{array}\right) .
$$

The six orderings applied to the six diagrams in Fig. 1 generate 36 different diagrams with the corresponding momentum labels. However, the six diagrams in Fig. 2 are unchanged upon using the six orderings. Therefore, 42 different diagrams with various momentum labels are needed to take into account the squared amplitudes.

Nine Lorentz-invariant variables are defined as

$$
\begin{aligned}
& s_{12}=\left(p_{1}+p_{2}\right)^{2}, \quad s_{23}=\left(p_{2}+p_{3}\right)^{2}, \quad s_{31}=\left(p_{3}+p_{1}\right)^{2} \\
& u_{12} \equiv u_{15}=\left(p_{1}-p_{5}\right)^{2}, \quad u_{13} \equiv u_{16}=\left(p_{1}-p_{6}\right)^{2} \\
& u_{21} \equiv u_{24}=\left(p_{2}-p_{4}\right)^{2}, \quad u_{23} \equiv u_{26}=\left(p_{2}-p_{6}\right)^{2} \\
& u_{31} \equiv u_{34}=\left(p_{3}-p_{4}\right)^{2}, \quad u_{32} \equiv u_{35}=\left(p_{3}-p_{5}\right)^{2}
\end{aligned}
$$

The squared amplitudes and interference terms are expressed in terms of these independent variables for the three-quark to three-quark scatterings. Our Fortran code includes the squared amplitudes summed over spin and color states of the three final quarks and averaged over the spin and color states of the three initial quarks. All interference terms of amplitudes of different diagrams are also calculated. The two interference terms of a diagram in Fig. 1 and a diagram in Fig. 2 cancel each other because the terms are pure imaginary. For example,

$$
\mathcal{M}_{\mathrm{A}-45}^{+} \mathcal{M}_{\mathrm{A} * 4(56)}+\mathcal{M}_{\mathrm{A}-45} \mathcal{M}_{\mathrm{A} * 4(56)}^{+}=0 .
$$

We present the individually squared amplitudes and some interference terms of the second class of diagrams in Fig. 2, which are not zero, as

$$
\begin{gathered}
\left|\mathcal{M}_{\mathrm{A}_{*}}\right|^{2}=3 \bar{A} /\left(w_{1}^{2} w_{2}^{2} w_{3}^{2}\right), \\
\mathcal{M}_{\mathrm{A}_{*}} \mathcal{M}_{\mathrm{A}_{* 4(56)}^{+}}^{+}=\frac{3}{2} \bar{B} /\left(u_{13} u_{21} u_{32} w_{1} w_{2} w_{3}\right), \\
\mathcal{M}_{\mathrm{A}_{*}} \mathcal{M}_{\mathrm{A}_{*(45) 6}}^{+}=\frac{3}{2} \bar{C} /\left(u_{12} u_{23} u_{31} w_{1} w_{2} w_{3}\right), \\
\left|\mathcal{M}_{\mathrm{A}_{* 45}}\right|^{2}=3 \bar{D} /\left(u_{12}^{2} u_{21}^{2} w_{3}^{2}\right),
\end{gathered}
$$




$$
\begin{gathered}
\mathcal{M}_{\mathrm{A}_{* 45}} \mathcal{M}_{\mathrm{A}_{* 56}}^{+}=-\frac{3}{2} \bar{E} /\left(u_{12} u_{21} u_{23} u_{32} w_{1} w_{3}\right), \\
\mathcal{M}_{\mathrm{A}_{* 45}} \mathcal{M}_{\mathrm{A}_{* 64}}^{+}=-\frac{3}{2} \bar{F} /\left(u_{12} u_{21} u_{13} u_{31} w_{2} w_{3}\right), \\
\left|\mathcal{M}_{\mathrm{A}_{* 56}}\right|^{2}=3 \bar{G} /\left(u_{23}^{2} u_{32}^{2} w_{1}^{2}\right), \\
\mathcal{M}_{\mathrm{A}_{* 56}} \mathcal{M}_{\mathrm{A}_{* 64}}^{+}=-\frac{3}{2} \bar{H} /\left(u_{23} u_{32} u_{13} u_{31} w_{1} w_{2}\right), \\
\left|\mathcal{M}_{\mathrm{A}_{* 64}}\right|^{2}=3 \bar{I} /\left(u_{13}^{2} u_{31}^{2} w_{2}^{2}\right), \\
\left|\mathcal{M}_{\mathrm{A}_{* 4(56)}}\right|^{2}=3 \bar{J} /\left(u_{13}^{2} u_{21}^{2} u_{32}^{2}\right), \\
\mathcal{M}_{\mathrm{A}_{* 4(56)}} \mathcal{M}_{\mathrm{A}_{*(45) 6}}^{+}=-\frac{3}{2} \bar{K} /\left(u_{13} u_{21} u_{32} u_{12} u_{23} u_{31}\right), \\
\left|\mathcal{M}_{\mathrm{A}_{*(45) 6}}\right|^{2}=3 \bar{L} /\left(u_{12}^{2} u_{23}^{2} u_{31}^{2}\right),
\end{gathered}
$$

where $w_{1}=s_{12}+s_{31}+u_{12}+u_{13}, w_{2}=s_{12}+s_{23}+u_{21}+u_{23}$ and $w_{3}=s_{31}+s_{23}+u_{31}+u_{32} . \bar{A}$ is a function of the nine Lorentz-invariant variables, $\bar{A}=\sum A\left(n_{1}, n_{2}, n_{3}, n_{4}, n_{5}, n_{6}, n_{7}, n_{8}, n_{9}\right)$ $\times s_{12}^{n_{1}} s_{23}^{n_{2}} s_{31}^{n_{3}} u_{12}^{n_{4}} u_{13}^{n_{5}} u_{21}^{n_{6}} u_{23}^{n_{7}} u_{31}^{n_{8}} u_{32}^{n_{9}}$ where the sum is over $n_{1}, n_{2}, n_{3}, n_{4}, n_{5}, n_{6}, n_{7}, n_{8}$ and $n_{9}$, which run from 0 to 4 and are restricted to give $n_{1}+n_{2}+n_{3}+n_{4}+n_{5}+n_{6}+n_{7}+$ $n_{8}+n_{9}=4$. The non-zero A's are tabulated to form the column under $A$ in Table 1 . $\bar{B}, \bar{C}, \bar{D}, \bar{E}, \bar{F}, \bar{G}, \bar{H}, \bar{I}, \bar{J}, \bar{K}$ and $\bar{L}$ are similarly defined and $B, C, D, E, F, G, H, I, J, K$ and $L$ can be found in Table 1 . The above interference terms give the other interference terms, for example, $\mathcal{M}_{\mathrm{A}_{*}}^{+} \mathcal{M}_{\mathrm{A}_{* 4(56)}}=\mathcal{M}_{\mathrm{A}_{*}} \mathcal{M}_{\mathrm{A}_{* 4(56)}}^{+}$.

\section{Thermalization of quark matter}

To study the thermalization of quark matter, these three-quark to three-quark scatterings are incorporated into a Boltzmann-type transport equation

$$
\begin{aligned}
\frac{\partial f_{1}}{\partial t} & +\overrightarrow{\mathrm{v}}_{1} \cdot \vec{\nabla}_{\vec{r}} f_{1} \\
= & -\frac{\mathrm{g}_{\mathrm{Q}}}{2 E_{1}} \int \frac{d^{3} p_{2}}{(2 \pi)^{3} 2 E_{2}} \frac{d^{3} p_{3}}{(2 \pi)^{3} 2 E_{3}} \frac{d^{3} p_{4}}{(2 \pi)^{3} 2 E_{4}}(2 \pi)^{4} \delta^{4}\left(p_{1}+p_{2}-p_{3}-p_{4}\right) \\
& \times\left(\frac{1}{2}\left|\mathcal{M}_{\mathrm{uu} \rightarrow \mathrm{uu}}\right|^{2}+\left|\mathcal{M}_{\mathrm{ud} \rightarrow \mathrm{ud}}\right|^{2}\right)\left[f_{1} f_{2}\left(1-f_{3}\right)\left(1-f_{4}\right)-f_{3} f_{4}\left(1-f_{1}\right)\left(1-f_{2}\right)\right] \\
& -\frac{\mathrm{g}_{\mathrm{Q}}^{2}}{2 E_{1}} \int \frac{d^{3} p_{2}}{(2 \pi)^{3} 2 E_{2}} \frac{d^{3} p_{3}}{(2 \pi)^{3} 2 E_{3}} \frac{d^{3} p_{4}}{(2 \pi)^{3} 2 E_{4}} \frac{d^{3} p_{5}}{(2 \pi)^{3} 2 E_{5}} \frac{d^{3} p_{6}}{(2 \pi)^{3} 2 E_{6}} \\
& \times(2 \pi)^{4} \delta^{4}\left(p_{1}+p_{2}+p_{3}-p_{4}-p_{5}-p_{6}\right) \\
& \times\left[\frac{1}{12}\left|\mathcal{M}_{\mathrm{uuu} \rightarrow \mathrm{uuu}}\right|^{2}+\frac{1}{4}\left(\left|\mathcal{M}_{\mathrm{uud} \rightarrow \mathrm{uud}}\right|^{2}+\left|\mathcal{M}_{\mathrm{udu} \rightarrow \mathrm{udu}}\right|^{2}\right)+\frac{1}{4}\left|\mathcal{M}_{\text {udd } \rightarrow \text { udd }}\right|^{2}\right] \\
& \times\left[f_{1} f_{2} f_{3}\left(1-f_{4}\right)\left(1-f_{5}\right)\left(1-f_{6}\right)-f_{4} f_{5} f_{6}\left(1-f_{1}\right)\left(1-f_{2}\right)\left(1-f_{3}\right)\right]
\end{aligned}
$$

where the degeneracy factor $\mathrm{g}_{\mathrm{Q}}=6$ and the velocity of a massless quark $\mathrm{v}_{1}=1$. The distribution function $f_{\mathrm{i}}$ depends on the position $\vec{r}_{\mathrm{i}}$, the momentum $\vec{p}_{\mathrm{i}}$ and the time $t$. The distribution function $f_{1}$ in this equation gives the up-quark distribution in quark matter. 
We assume that quark matter studied here has the same down-quark distribution as the up-quark distribution. The first term on the right-hand side of the above equation is for the 2-quark to 2-quark scatterings. The second term is a new term representing the 3-quark to 3-quark scatterings and involving six quark distributions.

The squared amplitude $\left|\mathcal{M}_{\text {uuu } \rightarrow \text { uuu }}\right|^{2}$ is for the scatterings of three identical quarks. It equals the sum of the individually squared amplitudes for the 42 diagrams discussed in the last section and the amplitudes corresponding to the interference terms between these diagrams. Both of the squared amplitudes, $\left|\mathcal{M}_{\text {uud } \rightarrow \text { uud }}\right|^{2}$ and $\left|\mathcal{M}_{\text {udu } \rightarrow \text { udu }}\right|^{2}$, are for the scatterings of two up-quarks and a down-quark. While the former receives contributions only from the diagrams $\mathrm{A}_{-}, \mathrm{A}_{-45}, \mathrm{~A}_{*}$ and $\mathrm{A}_{* 45}$, the latter has contributions only from the diagrams $\mathrm{A}_{-}, \mathrm{A}_{-64}, \mathrm{~A}_{*}$ and $\mathrm{A}_{* 64}$. The squared amplitude $\left|\mathcal{M}_{\text {udd } \rightarrow \text { udd }}\right|^{2}$ is for the scatterings of one up-quark and two down-quarks. Only the diagrams $\mathrm{A}_{-}, \mathrm{A}_{-56}, \mathrm{~A}_{*}$ and $\mathrm{A}_{* 56}$ contribute to the squared amplitude.

\section{Numerical results}

Anisotropic parton momentum distributions are formed in initial $\mathrm{Au}-\mathrm{Au}$ collisions. Such anisotropy can be eliminated by elastic scatterings among partons. Starting from the time $t_{\text {ini }}$, when anisotropic quark matter is formed and ending at the time $t_{\text {iso }}$, when local momentum isotropy is established, the transport equation is solved. The squared amplitudes are calculated at $\alpha_{\mathrm{s}}=0.6$. In the calculations, a divergence of the squared amplitudes is encountered. The divergence is removed while gluon propagators are regularized by a screening mass which is evaluated from the distribution function by a formula used in Refs. [17-19]. We arbitrarily add $100 \mathrm{MeV}$ to or subtract $100 \mathrm{MeV}$ from the screening mass, a change of thermalization time by about $40 \%$ is obtained. Any two runs of Fortran codes for solving the transport equation do not give rise to a difference of screening masses more than $100 \mathrm{MeV}$. Therefore, the uncertainty in the thermalization time due to the screening mass is less than $40 \%$.

Since the initial gluon rapidity density accounting for the RHIC data $[12,13]$ is about 1000, larger than 200 given by HIJING [22], the initial quark rapidity density must also be similarly underestimated by HIJING simulation. For a central Au-Au collision at $\sqrt{s_{N N}}=200 \mathrm{GeV}$, an initial quark momentum distribution can be, for example, four times [23] as large as that obtained from HIJING simulation [24],

$$
f\left(\vec{p}, t_{\text {ini }}\right)=\frac{7.12 \times 10^{5}(2 \pi)^{1.5}}{\pi R_{A}^{2} Y(|\vec{p}| / \cosh (\mathrm{y})+0.3)} \mathrm{e}^{-|\vec{p}| /(0.9 \cosh (\mathrm{y}))-(|\vec{p}| \tanh (\mathrm{y}))^{2} / 8} \bar{\theta}\left(Y^{2}-\mathrm{y}^{2}\right)
$$

where $|\vec{p}|$ is in $\mathrm{GeV}$, the nuclear radius $R_{A}=6.4 \mathrm{fm}, t_{\text {ini }}=0.2 \mathrm{fm} / c$ and the rapidity y is less than $Y$ which approximately equals $5 . \bar{\theta}(x)$ is the step function which is zero for $x<0$ or 1 for $x \geq 0$. Anisotropy of the distribution is shown in Fig. 3 by the differences of dotted, dashed and dot-dashed curves, which represent the quark momentum distributions at the three angles $\theta=0^{\circ}, 45^{\circ}, 90^{\circ}$ relative to one incoming gold beam direction. The quark momentum distribution in the longitudinal direction is considerably larger than one in the transverse direction when momentum departs from 0.

Numerical simulations of the $2 \rightarrow 2$ and $3 \rightarrow 3$ scatterings in Fortran codes depend

on quark positions. Starting from the cross section for the 2-quark to 2-quark scattering $\sigma_{2 \rightarrow 2}$ which is calculated from the squared amplitudes in Eqs. (1)-(2), a scattering of two 
quarks is considered to occur when the closest distance of the two quarks is less than an interaction range of $\sqrt{\sigma_{2 \rightarrow 2} / \pi}$. Regarding to a three-quark scattering, the three quarks must be in a sphere which center is at the center-of-mass of the three quarks and which radius $r_{\mathrm{hs}}$ is determined by [11]

$$
\begin{aligned}
\pi r_{\mathrm{hs}}^{2}= & \frac{1}{3 !} \int \frac{d^{3} p_{4}}{(2 \pi)^{3} 2 E_{4}} \frac{d^{3} p_{5}}{(2 \pi)^{3} 2 E_{5}} \frac{d^{3} p_{6}}{(2 \pi)^{3} 2 E_{6}} \\
& \times(2 \pi)^{4} \delta^{4}\left(p_{1}+p_{2}+p_{3}-p_{4}-p_{5}-p_{6}\right)\left|\mathcal{M}_{3 \rightarrow 3}\right|^{2}
\end{aligned}
$$

where $\left|\mathcal{M}_{3 \rightarrow 3}\right|^{2}$ is the squared amplitude for the 3-quark to 3-quark scattering. One consequence of such numerical simulations is to unfortunately break the locality of the transport equation and thus Lorentz covariance. Nevertheless, the particle subdivision technique [20,21] can restore the Lorentz covariance [11] by means of the transformation

$$
\begin{gathered}
f \rightarrow f^{\prime}=\ell f, \\
\left|\mathcal{M}_{2 \rightarrow 2}\right|^{2} \rightarrow\left|\mathcal{M}_{2 \rightarrow 2}^{\prime}\right|^{2}=\left|\mathcal{M}_{2 \rightarrow 2}\right|^{2} / \ell, \quad\left|\mathcal{M}_{3 \rightarrow 3}\right|^{2} \rightarrow\left|\mathcal{M}_{3 \rightarrow 3}^{\prime}\right|^{2}=\left|\mathcal{M}_{3 \rightarrow 3}\right|^{2} / \ell^{2},
\end{gathered}
$$

in combination with the replacement

$$
\begin{gathered}
{\left[f_{1} f_{2}\left(1-f_{3}\right)\left(1-f_{4}\right)-f_{3} f_{4}\left(1-f_{1}\right)\left(1-f_{2}\right)\right] \rightarrow f_{1} f_{2}-f_{3} f_{4}} \\
{\left[f_{1} f_{2} f_{3}\left(1-f_{4}\right)\left(1-f_{5}\right)\left(1-f_{6}\right)-f_{4} f_{5} f_{6}\left(1-f_{1}\right)\left(1-f_{2}\right)\left(1-f_{3}\right)\right] \rightarrow f_{1} f_{2} f_{3}-f_{4} f_{5} f_{6}}
\end{gathered}
$$

In principle, the Lorentz covariance is recovered while $\ell$ goes to infinity. The practical value of $\ell$ in codes equals 40. Six hundred and sixty-six quarks are generated from the initial distribution (5) and the average of 20 runs of Fortran codes with different random number sets is taken as a solution of Eq. (4).

The solution of the transport equation at $t_{\text {iso }}=2 \mathrm{fm} / c$ is shown by the dotted, dashed and dot-dashed curves in Fig. 4. The curves overlap and can thus be fitted to the Jüttner distribution,

$$
f\left(\vec{p}, t_{\text {iso }}\right)=\frac{\lambda}{\mathrm{e}^{|\vec{p}| / T}-\lambda}
$$

where the temperature of quark matter $T=0.59 \mathrm{GeV}$ and fugacity $\lambda=0.04$. We get a thermalization time of $t_{\text {iso }}-t_{\text {ini }}=1.8 \mathrm{fm} / c$. For strongly interacting quark matter, the present value of the coupling constant $\alpha_{\mathrm{s}}=0.6$ is allowed [25]. For weakly interacting quark matter, $\alpha_{\mathrm{s}}$ needs to be reduced. If the initial quark distribution and/or the coupling constant becomes smaller, the thermalization time of quark matter gets longer than the order of $1.8 \mathrm{fm} / c$.

\section{Summary}

We have studied the thermalization of quark matter including three-quark elastic scatterings in the transport equation. Squared amplitudes for triple-quark scatterings are derived at the tree level in QCD. Given an anisotropic quark distribution at the formation of quark matter, momentum isotropy can be shown by the transport equation solution. The history of quark matter's evolving into a thermal state can be described by the transport equation. The triple-quark scatterings give a variation of quark distribution function 
comparable to the one that resulted from the quark-quark scatterings. Rapid thermalization can not be established by the triple-quark scatterings from such an anisotropic quark distribution which can appropriately describe quark matter initially created in central Au-Au collisions at the highest RHIC energy. Therefore, we also conclude that rapid thermalization of quark matter must depend on the gluon-gluon-quark, gluon-quark-quark and gluon-antiquark-quark scatterings which involve higher gluon number density than the quark number density and have larger squared amplitudes than the quark-quarkquark scatterings. Such very complicated works including the quark scatterings with gluons and/or antiquarks remain to be done.

\section{Acknowledgements}

This work was supported in part by National Natural Science Foundation of China under Grant No. 10135030, in part by Shanghai Education Committee Research Fund and in part by the CAS Knowledge Innovation Project No. KJCX2-SW-N02. 


\section{References}

[1]U. Heinz, P. F. Kolb, in: R. Bellwied, J. Harris, W. Bauer (Eds.), Proc. of the 18th Winter Workshop on Nuclear Dynamics, EP Systema, Debrecen, Hungary, 2002.

[2]E. V. Shuryak, Nucl. Phys. A715(2003)289c.

[3]T. Hirano, Phys. Rev. C65(2001)011901.

[4]P. Huovinen, Nucl. Phys. A715(2003)299c.

[5]K. Morita, S. Muroya, C. Nonaka, T. Hirano, Phys. Rev. C66(2002)054904.

[6]D. Teaney, J. Lauret, E. V. Shuryak, nucl-th/0110037.

[7]K. J. Eskola, H. Niemi, P. V. Ruuskanen, S. S. Räsänen, Phys. Lett. B566(2003)187;

K. J. Eskola, H. Niemi, P. V. Ruuskanen, S. S. Räsänen, Nucl. Phys. A715(2003)561c.

[8]K. H. Ackermann, et al., STAR Collaboration, Phys. Rev. Lett. 86(2001)402;

R. J. Snellings, et al., for the STAR Collaboration, Nucl. Phys. A698(2002)193c;

C. Adler, et al., STAR Collaboration, Phys. Rev. Lett. 87(2001)182301;

C. Adler, et al., STAR Collaboration, Phys. Rev. C66(2002)034904;

C. Adler, et al., STAR Collaboration, Phys. Rev. Lett. 90(2003)032301.

[9]R. A. Lacey, et al., for the PHENIX Collaboration, Nucl. Phys. A698(2002)559c;

S. S. Adler, et al., PHENIX Collaboration, Phys. Rev. Lett. 91(2003)182301;

S. S. Adler, et al., PHENIX Collaboration, nucl-ex/0411040.

[10]K. Geiger, Phys. Rev. D46(1992)4965;

K. Geiger, Phys. Rev. D46(1992) 4986.

[11]X.-M. Xu, Y. Sun, A.-Q. Chen, L. Zheng, Nucl. Phys. A744(2004)347.

[12]K. J. Eskola, K. Kajantie, K. Tuominen, Phys. Lett. B497 (2001)39.

[13]M. Gyulassy, P. Lévai, I. Vitev, Nucl. Phys. B594(2001)371;

M. Gyulassy, I. Vitev, X.-N. Wang, P. Huovinen, Phys. Lett. B526(2001)301.

[14]F. Cooper, E. Mottola, G. C. Nayak, Phys. Lett. B555(2003) 181.

[15]R. Cutler, D. Sivers, Phys. Rev. D17(1978)196.

[16]B. L. Combridge, J. Kripfganz, J. Ranft, Phys. Lett. B70(1977)234.

[17]T. S. Biró, B. Müller, X.-N. Wang, Phys. Lett. B283(1992)171.

[18]K. J. Eskola, B. Müller, X.-N. Wang, Phys. Lett. B374(1996)20.

[19]S.A. Bass, B. Müller, D. K. Srivastava, Phys. Lett. B551(2003)277.

[20]B. Zhang, M. Gyulassy, Y. Pang, Phys. Rev. C58(1998)1175.

[21]D. Molnár, M. Gyulassy, Nucl. Phys. A697(2002)495.

[22]X.-N. Wang, M. Gyulassy, Phys. Rev. D44(1991)3501;

X.-N. Wang, M. Gyulassy, Comput. Phys. Commun. 83(1994)307;

X.-N. Wang, Phys. Rep. 280(1997)287.

[23]X.-M. Xu, D. Kharzeev, H. Satz, X.-N. Wang, Phys. Rev. C53 (1996)3051.

[24]P. Lévai, B. Müller, X.-N. Wang, Phys. Rev. C51(1995)3326.

[25]L.-W. Chen, C.M. Ko, Z.-W. Lin, Phys. Rev. C69(2004)031901. 


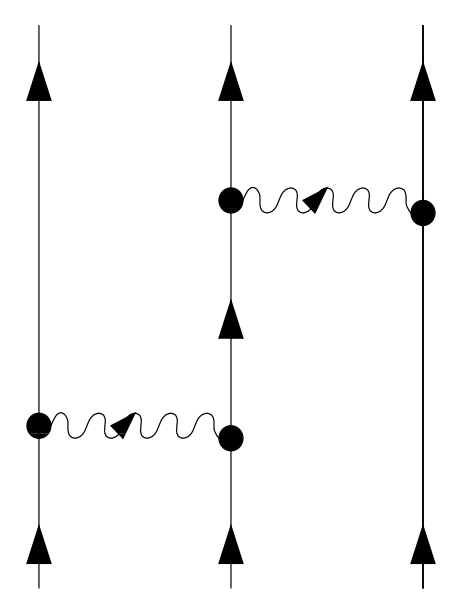

A

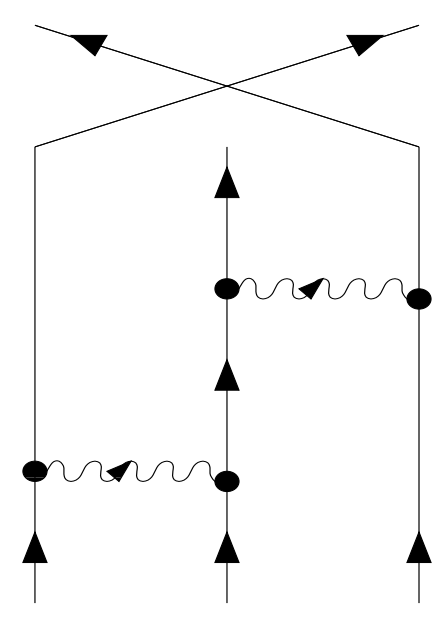

$\mathrm{A}_{-64}$

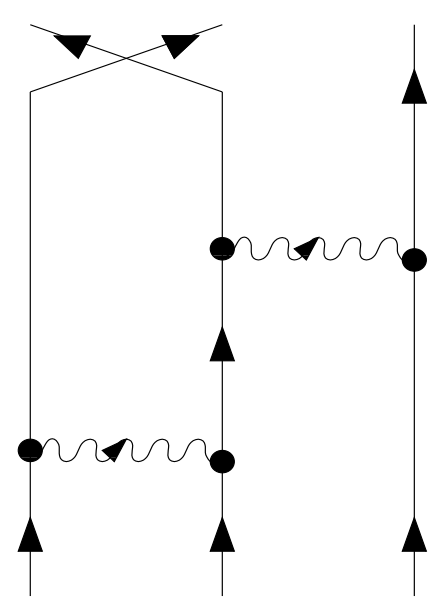

$\mathrm{A}_{-45}$

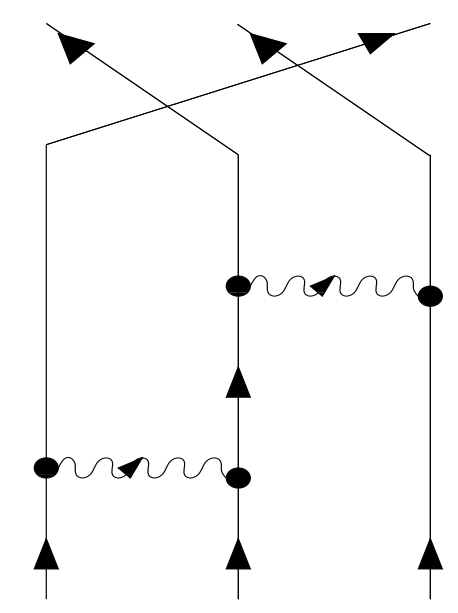

$\mathrm{A}_{-4(56)}$

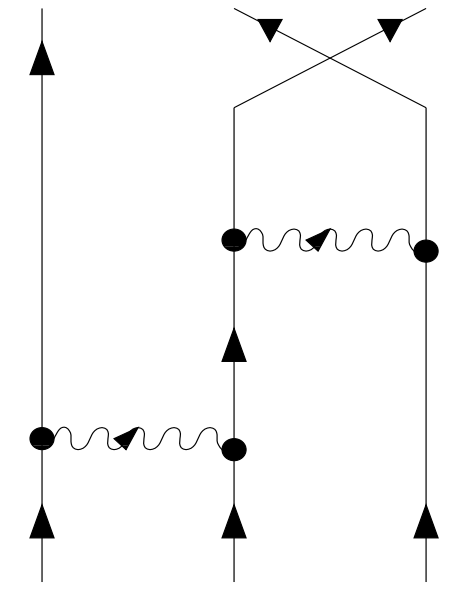

$\mathrm{A}_{-56}$

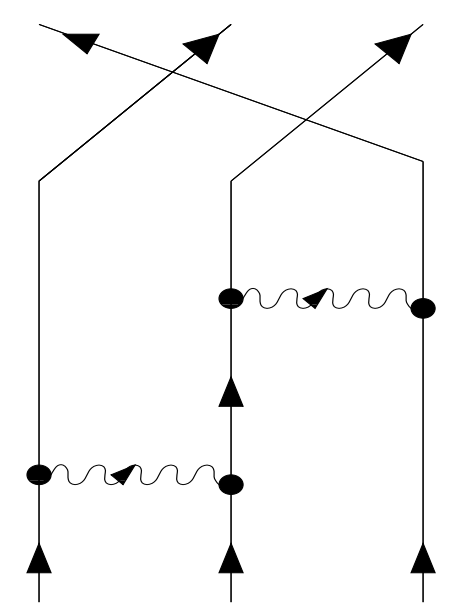

$\mathrm{A}_{-(45) 6}$

Figure 1: Two-gluon-exchange induced scatterings of three quarks. 


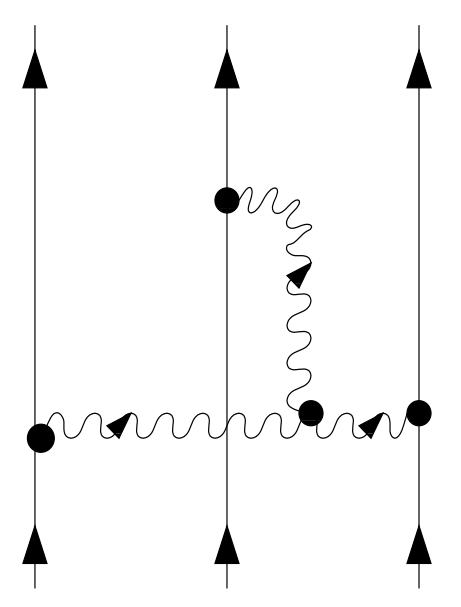

A*

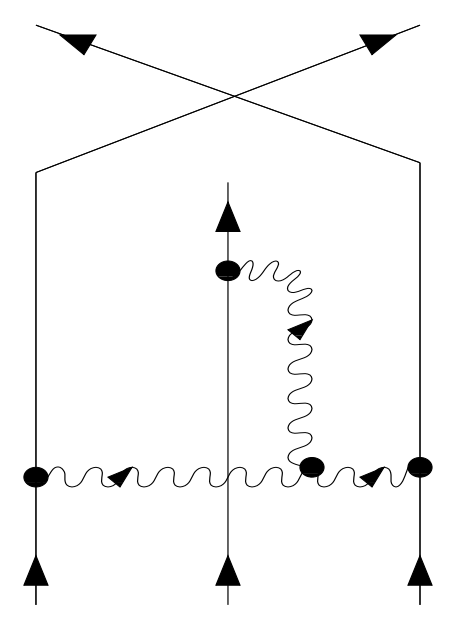

$\mathrm{A}_{* 64}$

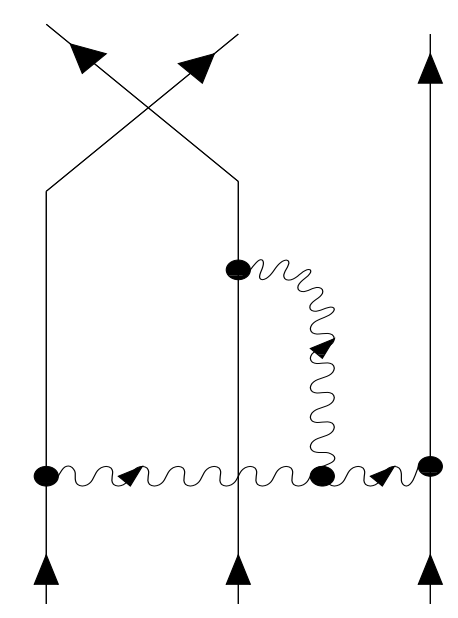

$\mathrm{A}_{* 45}$

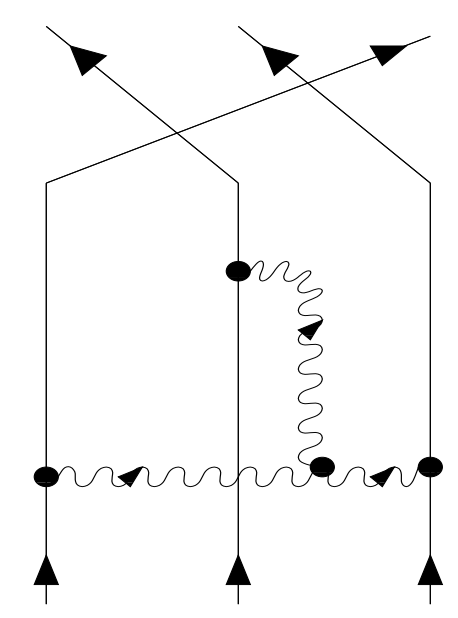

$\mathrm{A}_{* 4(56)}$

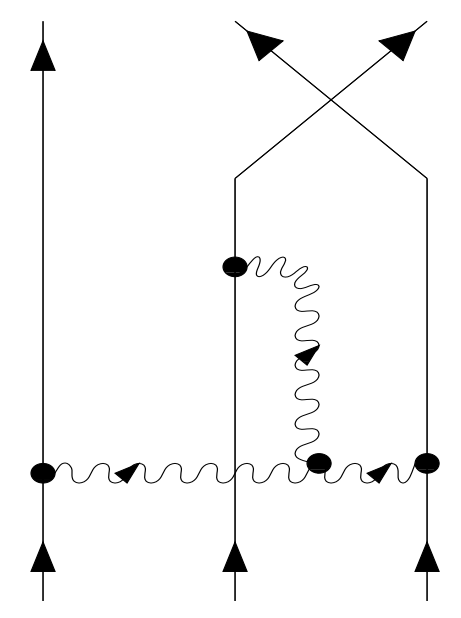

$\mathrm{A}_{* 56}$

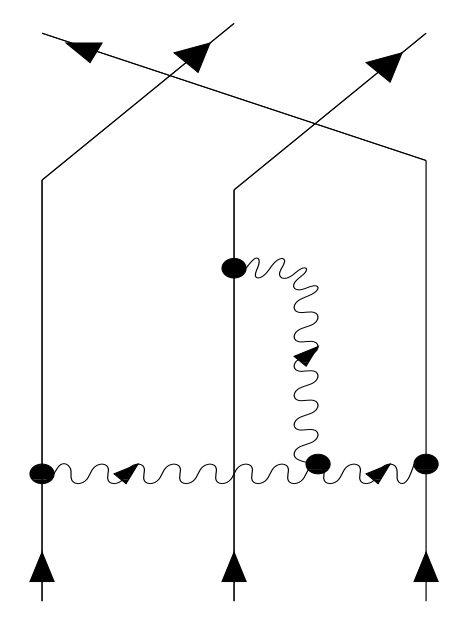

$\mathrm{A}_{*(45) 6}$

Figure 2: Triple-gluon coupling in scatterings of three quarks. 


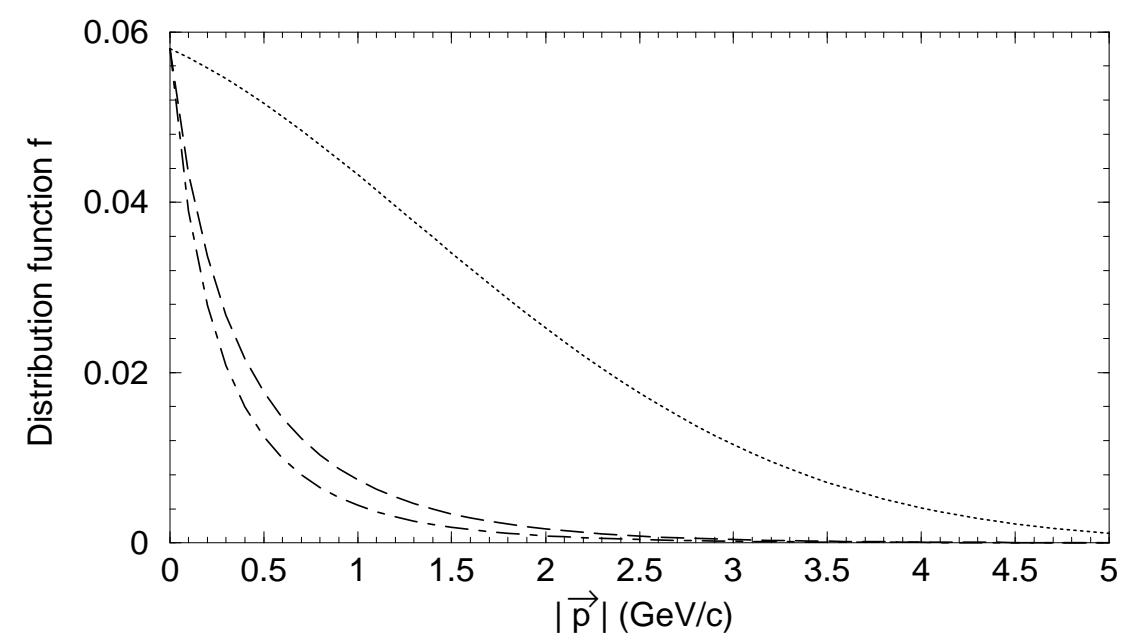

Figure 3: Quark distribution functions versus momentum in different directions while anisotropic quark matter is produced in the initial $\mathrm{Au}-\mathrm{Au}$ collision. The dotted, dashed and dot-dashed curves correspond to the angles $\theta=0^{\circ}, 45^{\circ}, 90^{\circ}$, respectively.

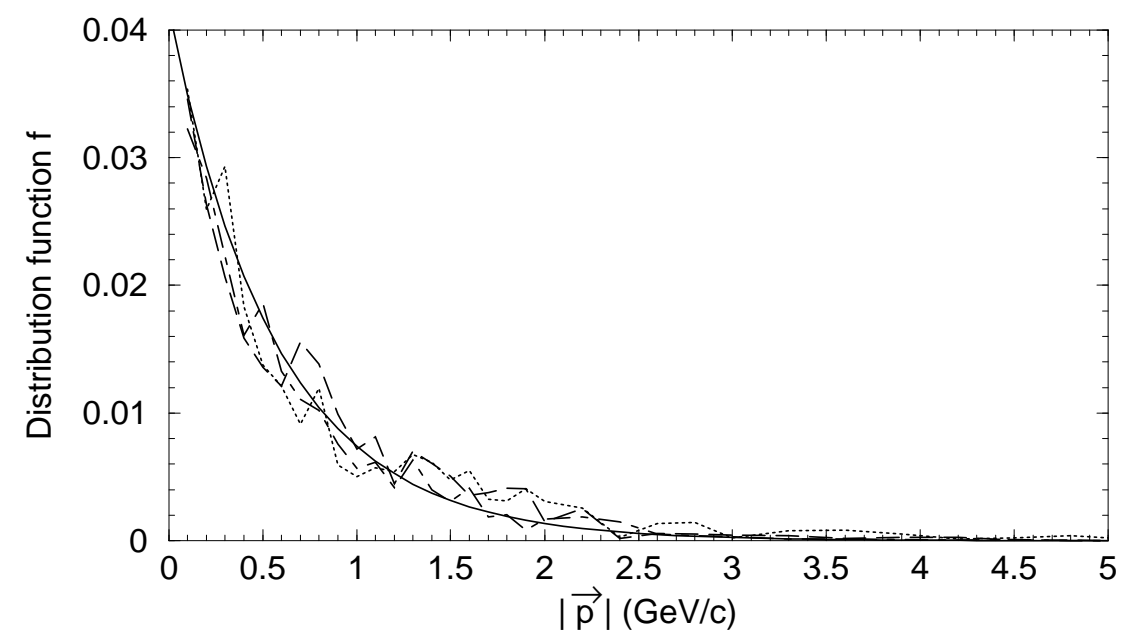

Figure 4: Quark distribution functions versus momentum in different directions while quark matter just arrives at thermal equilibrium. The dotted, dashed and dot-dashed curves correspond to the angles $\theta=0^{\circ}, 45^{\circ}, 90^{\circ}$, respectively. The solid curve represents the thermal distribution function. 
Table 1: For the entries we use the symbols $\left(a_{1}, \cdots, a_{23}\right)=(1024,512,2560,1536,2048$, 3072, 128, 256, 384, 768, 640, 896, 1152, 1280, 2304, 1792, 3328, 4096, 64, 448, 320, 192, $832) / 3456$.

\begin{tabular}{|c|c|c|c|c|c|c|c|c|c|c|c|c|}
\hline & $\overline{A A}$ & 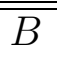 & $\overline{C C}$ & 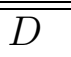 & $\overline{E E}$ & $\overline{F F}$ & $\overline{\bar{G}}$ & 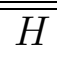 & 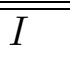 & 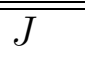 & 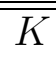 & 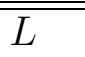 \\
\hline$u_{13} u_{23} u_{32}^{2}$ & 0 & 0 & $\overline{0}$ & $-a_{2}$ & 0 & 0 & $-a_{1}$ & 0 & $-a_{1}$ & $-a_{10}$ & 0 & 0 \\
\hline$u_{13} u_{23} u_{31} u_{32}$ & $-a_{1}$ & 0 & 0 & $-a_{1}$ & 0 & 0 & $-a_{4}$ & 0 & $-a_{4}$ & $-a_{10}$ & 0 & $-a_{8}$ \\
\hline$u_{13} u_{23} u_{31}^{2}$ & 0 & 0 & 0 & $-a_{2}$ & 0 & 0 & $-a_{1}$ & 0 & $-a_{1}$ & $-a_{2}$ & 0 & $-a_{8}$ \\
\hline$u_{13} u_{23}^{2} u_{32}$ & $a_{1}$ & 0 & 0 & 0 & 0 & 0 & 0 & 0 & $a_{2}$ & $a_{1}$ & 0 & $a_{8}$ \\
\hline$u_{13} u_{23}^{2} u_{31}$ & $a_{1}$ & 0 & 0 & 0 & 0 & 0 & $a_{2}$ & 0 & $a_{1}$ & $a_{2}$ & 0 & $a_{8}$ \\
\hline$u_{13} u_{21} u_{32}^{2}$ & $a_{2}$ & 0 & 0 & 0 & 0 & 0 & 0 & 0 & $-a_{1}$ & 0 & 0 & 0 \\
\hline$u_{13} u_{21} u_{31} u_{32}$ & $a_{2}$ & 0 & 0 & $a_{2}$ & 0 & 0 & $-a_{2}$ & 0 & $-a_{1}$ & $a_{8}$ & 0 & $-a_{8}$ \\
\hline$u_{13} u_{21} u_{31}^{2}$ & $a_{1}$ & 0 & 0 & $a_{2}$ & 0 & 0 & 0 & 0 & 0 & $a_{2}$ & 0 & 0 \\
\hline$u_{13} u_{21} u_{23} u_{32}$ & $a_{2}$ & 0 & 0 & $-a_{2}$ & 0 & 0 & $-a_{1}$ & 0 & $a_{2}$ & $a_{10}$ & 0 & $a_{8}$ \\
\hline$u_{13} u_{21} u_{23} u_{31}$ & $a_{1}$ & 0 & 0 & $-a_{2}$ & 0 & 0 & $-a_{2}$ & 0 & $a_{1}$ & 0 & 0 & 0 \\
\hline$u_{13} u_{21}^{2} u_{32}$ & $a_{2}$ & 0 & 0 & 0 & 0 & 0 & $-a_{1}$ & 0 & 0 & $a_{2}$ & 0 & 0 \\
\hline$u_{13} u_{21}^{2} u_{31}$ & $a_{1}$ & 0 & 0 & $a_{2}$ & 0 & 0 & 0 & 0 & $a_{1}$ & $a_{2}$ & 0 & 0 \\
\hline$u_{13}^{2} u_{23} u_{32}$ & $a_{1}$ & 0 & 0 & 0 & 0 & 0 & $a_{1}$ & 0 & $a_{2}$ & $a_{8}$ & 0 & $-a_{2}$ \\
\hline$u_{13}^{2} u_{23} u_{31}$ & $a_{1}$ & 0 & 0 & 0 & 0 & 0 & $a_{2}$ & 0 & 0 & $a_{1}$ & 0 & $a_{2}$ \\
\hline$u_{13}^{2} u_{21} u_{32}$ & $a_{2}$ & 0 & 0 & $-a_{1}$ & 0 & 0 & 0 & 0 & 0 & $a_{2}$ & 0 & $-a_{8}$ \\
\hline$u_{13}^{2} u_{21} u_{31}$ & 0 & 0 & 0 & $-a_{1}$ & 0 & 0 & $-a_{2}$ & 0 & $-a_{1}$ & 0 & 0 & $a_{8}$ \\
\hline$u_{12} u_{23} u_{32}^{2}$ & $a_{1}$ & 0 & 0 & $a_{2}$ & 0 & 0 & 0 & 0 & 0 & $a_{8}$ & 0 & $a_{1}$ \\
\hline$u_{12} u_{23} u_{31} u_{32}$ & $a_{2}$ & 0 & 0 & $a_{2}$ & 0 & 0 & $-a_{1}$ & 0 & $-a_{2}$ & $a_{8}$ & 0 & $a_{10}$ \\
\hline$u_{12} u_{23} u_{31}^{2}$ & $a_{2}$ & 0 & 0 & 0 & 0 & 0 & $-a_{1}$ & 0 & 0 & 0 & 0 & $a_{2}$ \\
\hline$u_{12} u_{23}^{2} u_{32}$ & 0 & 0 & 0 & $-a_{1}$ & 0 & 0 & $-a_{1}$ & 0 & $-a_{2}$ & 0 & 0 & $-a_{10}$ \\
\hline$u_{12} u_{23}^{2} u_{31}$ & $a_{2}$ & 0 & 0 & $-a_{1}$ & 0 & 0 & 0 & 0 & 0 & 0 & 0 & 0 \\
\hline$u_{12} u_{21} u_{32}^{2}$ & $a_{1}$ & 0 & 0 & $a_{1}$ & 0 & 0 & $a_{2}$ & 0 & 0 & $a_{8}$ & 0 & $a_{2}$ \\
\hline$u_{12} u_{21} u_{31} u_{32}$ & $a_{1}$ & 0 & 0 & $a_{1}$ & 0 & 0 & $-a_{2}$ & 0 & $-a_{2}$ & 0 & 0 & 0 \\
\hline$u_{12} u_{21} u_{31}^{2}$ & $a_{1}$ & 0 & 0 & $a_{1}$ & 0 & 0 & 0 & 0 & $a_{2}$ & 0 & 0 & $a_{2}$ \\
\hline$u_{12} u_{21} u_{23} u_{32}$ & $-a_{1}$ & 0 & 0 & $-a_{4}$ & 0 & 0 & $-a_{4}$ & 0 & $-a_{1}$ & $-a_{8}$ & 0 & $-a_{10}$ \\
\hline$u_{12} u_{21} u_{23} u_{31}$ & $a_{2}$ & 0 & 0 & $-a_{1}$ & 0 & 0 & $-a_{2}$ & 0 & $a_{2}$ & $-a_{8}$ & 0 & $a_{8}$ \\
\hline$u_{12} u_{21}^{2} u_{32}$ & 0 & 0 & 0 & $-a_{1}$ & 0 & 0 & $-a_{1}$ & 0 & $-a_{2}$ & $-a_{8}$ & 0 & $-a_{2}$ \\
\hline$u_{12} u_{21}^{2} u_{31}$ & $a_{1}$ & 0 & 0 & 0 & 0 & 0 & 0 & 0 & $a_{2}$ & 0 & 0 & $a_{2}$ \\
\hline$u_{12} u_{13} u_{23} u_{32}$ & $a_{1}$ & 0 & 0 & $-a_{2}$ & 0 & 0 & $a_{1}$ & 0 & $-a_{2}$ & $-a_{14}$ & 0 & $-a_{14}$ \\
\hline$u_{12} u_{13} u_{23} u_{31}$ & $a_{2}$ & 0 & 0 & $-a_{2}$ & 0 & 0 & $a_{2}$ & 0 & $-a_{1}$ & $-a_{8}$ & 0 & $-a_{8}$ \\
\hline$u_{12} u_{13} u_{21} u_{32}$ & $a_{2}$ & 0 & 0 & $-a_{1}$ & 0 & 0 & $a_{2}$ & 0 & $-a_{2}$ & $-a_{8}$ & 0 & $-a_{8}$ \\
\hline$u_{12} u_{13} u_{21} u_{31}$ & $-a_{1}$ & 0 & 0 & $-a_{4}$ & 0 & 0 & $-a_{1}$ & 0 & $-a_{4}$ & $-a_{2}$ & 0 & $-a_{2}$ \\
\hline$u_{12}^{2} u_{23} u_{32}$ & $a_{1}$ & 0 & 0 & $a_{2}$ & 0 & 0 & $a_{1}$ & 0 & 0 & $-a_{2}$ & 0 & $a_{8}$ \\
\hline$u_{12}^{2} u_{23} u_{31}$ & $a_{2}$ & 0 & 0 & 0 & 0 & 0 & 0 & 0 & $-a_{1}$ & $-a_{8}$ & 0 & $a_{2}$ \\
\hline$u_{12}^{2} u_{21} u_{32}$ & $a_{1}$ & 0 & 0 & 0 & 0 & 0 & $a_{2}$ & 0 & 0 & $a_{2}$ & 0 & $a_{1}$ \\
\hline$u_{12}^{2} u_{21} u_{31}$ & 0 & 0 & 0 & $-a_{1}$ & 0 & 0 & $-a_{2}$ & 0 & $-a_{1}$ & $a_{8}$ & 0 & 0 \\
\hline
\end{tabular}


Table 1. Continued.

\begin{tabular}{|c|c|c|c|c|c|c|c|c|c|c|c|c|}
\hline & $\overline{A A}$ & $\overline{\bar{B}}$ & $\overline{C C}$ & $\overline{D D}$ & $\bar{E}$ & $\overline{F F}$ & 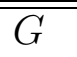 & 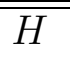 & $\overline{\overline{I I}}$ & 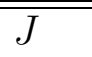 & $\bar{~} \bar{K}$ & 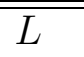 \\
\hline$s_{31} u_{23} u_{32}^{2}$ & $\overline{a_{2}}$ & 0 & 0 & $a_{2}$ & 0 & 0 & $-a_{2}$ & 0 & 0 & $-a_{8}$ & 0 & 0 \\
\hline$s_{31} u_{23} u_{31} u_{32}$ & 0 & 0 & 0 & 0 & 0 & 0 & $-a_{1}$ & 0 & $-a_{1}$ & $-a_{8}$ & 0 & $-a_{2}$ \\
\hline$s_{31} u_{23} u_{31}^{2}$ & $a_{2}$ & 0 & 0 & $-a_{2}$ & 0 & 0 & $-a_{1}$ & 0 & $-a_{2}$ & 0 & 0 & $-a_{8}$ \\
\hline$s_{31} u_{23}^{2} u_{32}$ & 0 & 0 & 0 & $-a_{1}$ & 0 & 0 & 0 & 0 & $-a_{1}$ & $a_{2}$ & 0 & $a_{2}$ \\
\hline$s_{31} u_{23}^{2} u_{31}$ & 0 & 0 & 0 & $-a_{1}$ & 0 & 0 & 0 & 0 & 0 & 0 & 0 & $a_{2}$ \\
\hline$s_{31} u_{23}^{3}$ & $a_{2}$ & 0 & 0 & 0 & 0 & 0 & $-a_{2}$ & 0 & $a_{2}$ & 0 & 0 & $-a_{8}$ \\
\hline$s_{31} u_{21} u_{32}^{2}$ & $a_{1}$ & 0 & 0 & $a_{2}$ & 0 & 0 & $a_{2}$ & 0 & 0 & 0 & 0 & 0 \\
\hline$s_{31} u_{21} u_{31} u_{32}$ & $a_{2}$ & 0 & 0 & $a_{2}$ & 0 & 0 & $a_{2}$ & 0 & 0 & 0 & 0 & $-a_{2}$ \\
\hline$s_{31} u_{21} u_{31}^{2}$ & $a_{2}$ & 0 & 0 & 0 & 0 & 0 & 0 & 0 & $a_{2}$ & 0 & 0 & 0 \\
\hline$s_{31} u_{21} u_{23} u_{32}$ & $-a_{2}$ & 0 & 0 & $-a_{2}$ & 0 & 0 & $-a_{2}$ & 0 & $-a_{1}$ & $a_{10}$ & 0 & $a_{2}$ \\
\hline$s_{31} u_{21} u_{23} u_{31}$ & $a_{1}$ & 0 & 0 & $-a_{4}$ & 0 & 0 & $-a_{4}$ & 0 & 0 & $a_{8}$ & 0 & $a_{2}$ \\
\hline$s_{31} u_{21} u_{23}^{2}$ & $a_{2}$ & 0 & 0 & $-a_{1}$ & 0 & 0 & $-a_{1}$ & 0 & $a_{2}$ & $-a_{2}$ & 0 & $-a_{2}$ \\
\hline$s_{31} u_{21}^{2} u_{32}$ & $-a_{2}$ & 0 & 0 & 0 & 0 & 0 & 0 & 0 & 0 & $a_{8}$ & 0 & 0 \\
\hline$s_{31} u_{21}^{2} u_{31}$ & $a_{1}$ & 0 & 0 & $-a_{2}$ & 0 & 0 & 0 & 0 & $a_{1}$ & 0 & 0 & $a_{2}$ \\
\hline$s_{31} u_{21}^{2} u_{23}$ & $a_{2}$ & 0 & 0 & $-a_{1}$ & 0 & 0 & $-a_{1}$ & 0 & $a_{2}$ & $-a_{2}$ & 0 & $-a_{2}$ \\
\hline$s_{31} u_{21}^{3}$ & $a_{2}$ & 0 & 0 & $-a_{2}$ & 0 & 0 & 0 & 0 & $a_{2}$ & $-a_{8}$ & 0 & 0 \\
\hline$s_{31} u_{13} u_{23} u_{32}$ & $a_{1}$ & 0 & 0 & 0 & 0 & 0 & $a_{2}$ & 0 & $-a_{1}$ & $-a_{8}$ & 0 & $-a_{1}$ \\
\hline$s_{31} u_{13} u_{23} u_{31}$ & 0 & $-a_{7}$ & $-a_{7}$ & 0 & 0 & 0 & $a_{2}$ & 0 & $-a_{5}$ & $a_{14}$ & $a_{7}$ & $a_{14}$ \\
\hline$s_{31} u_{13} u_{23}^{2}$ & $a_{1}$ & 0 & 0 & 0 & 0 & 0 & $-a_{2}$ & 0 & $a_{1}$ & $a_{2}$ & 0 & $a_{10}$ \\
\hline$s_{31} u_{13} u_{21} u_{32}$ & $a_{2}$ & 0 & 0 & 0 & 0 & 0 & 0 & 0 & $-a_{5}$ & $a_{10}$ & 0 & $-a_{1}$ \\
\hline$s_{31} u_{13} u_{21} u_{31}$ & 0 & $-a_{7}$ & $-a_{7}$ & $a_{2}$ & 0 & 0 & 0 & 0 & $-a_{5}$ & $a_{4}$ & $a_{7}$ & $a_{10}$ \\
\hline$s_{31} u_{13} u_{21} u_{23}$ & $a_{1}$ & $-a_{7}$ & $-a_{7}$ & $-a_{4}$ & 0 & 0 & $-a_{4}$ & 0 & 0 & $a_{1}$ & $a_{7}$ & $a_{15}$ \\
\hline$s_{31} u_{13} u_{21}^{2}$ & 0 & $-a_{7}$ & $-a_{7}$ & 0 & 0 & 0 & $-a_{1}$ & 0 & 0 & $a_{1}$ & $a_{7}$ & $a_{1}$ \\
\hline$s_{31} u_{13}^{2} u_{23}$ & $a_{2}$ & $a_{7}$ & $a_{7}$ & 0 & 0 & 0 & 0 & 0 & $a_{2}$ & $-a_{2}$ & $-a_{7}$ & $-a_{2}$ \\
\hline$s_{31} u_{13}^{2} u_{21}$ & $a_{2}$ & $a_{7}$ & $a_{7}$ & $-a_{1}$ & 0 & 0 & $-a_{2}$ & 0 & $-a_{2}$ & $-a_{10}$ & $-a_{7}$ & $-a_{8}$ \\
\hline$s_{31} u_{12} u_{23} u_{32}$ & $a_{3}$ & 0 & 0 & $a_{4}$ & 0 & 0 & $a_{1}$ & 0 & 0 & $a_{1}$ & 0 & $a_{2}$ \\
\hline$s_{31} u_{12} u_{23} u_{31}$ & $a_{2}$ & $-a_{7}$ & $-a_{7}$ & 0 & 0 & 0 & 0 & 0 & $-a_{5}$ & $a_{2}$ & $a_{7}$ & $a_{16}$ \\
\hline$s_{31} u_{12} u_{23}^{2}$ & $-a_{2}$ & 0 & 0 & 0 & 0 & 0 & 0 & 0 & 0 & $-a_{1}$ & 0 & $a_{8}$ \\
\hline$s_{31} u_{12} u_{21} u_{32}$ & $a_{3}$ & 0 & 0 & $a_{1}$ & 0 & 0 & $a_{4}$ & 0 & 0 & $a_{2}$ & 0 & $a_{4}$ \\
\hline$s_{31} u_{12} u_{21} u_{31}$ & $a_{1}$ & 0 & 0 & $a_{2}$ & 0 & 0 & 0 & 0 & $-a_{1}$ & $a_{2}$ & 0 & $a_{4}$ \\
\hline$s_{31} u_{12} u_{21} u_{23}$ & $-a_{2}$ & $-a_{7}$ & $-a_{7}$ & $-a_{2}$ & 0 & 0 & $-a_{2}$ & 0 & $-a_{1}$ & 0 & $a_{7}$ & $a_{14}$ \\
\hline$s_{31} u_{12} u_{21}^{2}$ & 0 & 0 & 0 & 0 & 0 & 0 & $-a_{1}$ & 0 & $-a_{1}$ & $a_{2}$ & 0 & $a_{1}$ \\
\hline$s_{31} u_{12} u_{13} u_{23}$ & $a_{2}$ & $a_{8}$ & $a_{8}$ & $a_{2}$ & 0 & 0 & $a_{2}$ & 0 & 0 & $-a_{5}$ & $-a_{8}$ & $-a_{16}$ \\
\hline$s_{31} u_{12} u_{13} u_{21}$ & 0 & $a_{7}$ & $a_{7}$ & $-a_{1}$ & 0 & 0 & 0 & 0 & $-a_{1}$ & $-a_{4}$ & $-a_{7}$ & $-a_{15}$ \\
\hline$s_{31} u_{12}^{2} u_{23}$ & $a_{1}$ & $a_{7}$ & $a_{7}$ & $a_{2}$ & 0 & 0 & $a_{2}$ & 0 & 0 & 0 & $-a_{7}$ & $-a_{2}$ \\
\hline$s_{31} u_{12}^{2} u_{21}$ & $a_{2}$ & 0 & 0 & $-a_{2}$ & 0 & 0 & $a_{2}$ & 0 & 0 & 0 & 0 & $-a_{2}$ \\
\hline$s_{31}^{2} u_{23} u_{32}$ & $a_{1}$ & $a_{8}$ & $-a_{8}$ & 0 & $a_{8}$ & $a_{8}$ & 0 & $-a_{8}$ & 0 & $-a_{2}$ & $a_{7}$ & 0 \\
\hline$s_{31}^{2} u_{23} u_{31}$ & 0 & $a_{7}$ & $-a_{9}$ & $-a_{1}$ & 0 & $a_{2}$ & 0 & 0 & $-a_{5}$ & $a_{10}$ & $-a_{7}$ & $a_{4}$ \\
\hline$s_{31}^{2} u_{23}^{2}$ & 0 & $-a_{8}$ & $a_{8}$ & $a_{1}$ & $-a_{8}$ & $-a_{8}$ & 0 & $a_{8}$ & 0 & $a_{1}$ & $-a_{7}$ & $a_{2}$ \\
\hline$s_{31}^{2} u_{21} u_{32}$ & $a_{5}$ & $-a_{7}$ & $-a_{8}$ & 0 & $a_{2}$ & 0 & 0 & 0 & 0 & 0 & $a_{7}$ & 0 \\
\hline
\end{tabular}


Table 1. Continued.

\begin{tabular}{|c|c|c|c|c|c|c|c|c|c|c|c|c|}
\hline & $\overline{\bar{A} A}$ & $\overline{\overline{B B}}$ & 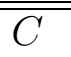 & 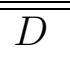 & $\overline{\bar{E}}$ & $\overline{F F}$ & $\overline{\bar{G}}$ & 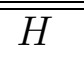 & 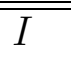 & 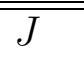 & 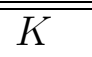 & 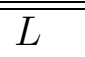 \\
\hline$s_{31}^{2} u_{21} u_{31}$ & $a_{1}$ & 0 & $-a_{2}$ & $-a_{2}$ & $a_{8}$ & $a_{8}$ & $a_{2}$ & $-a_{8}$ & 0 & $a_{8}$ & 0 & $a_{4}$ \\
\hline$s_{31}^{2} u_{21} u_{23}$ & $-a_{1}$ & 0 & $a_{7}$ & $a_{2}$ & $-a_{10}$ & $a_{8}$ & $a_{2}$ & $a_{8}$ & $-a_{5}$ & $a_{5}$ & $-a_{9}$ & $a_{3}$ \\
\hline$s_{31}^{2} u_{21}^{2}$ & 0 & $a_{7}$ & $-a_{8}$ & 0 & $-a_{8}$ & $a_{8}$ & $a_{1}$ & $-a_{8}$ & 0 & $a_{2}$ & $-a_{7}$ & $a_{5}$ \\
\hline$s_{31}^{2} u_{13} u_{23}$ & $a_{1}$ & $-a_{8}$ & $a_{7}$ & $a_{2}$ & $a_{8}$ & $-a_{8}$ & $-a_{2}$ & $a_{8}$ & 0 & 0 & $-a_{7}$ & $-a_{1}$ \\
\hline$s_{31}^{2} u_{13} u_{21}$ & 0 & $-a_{7}$ & $a_{8}$ & 0 & 0 & 0 & $-a_{1}$ & $a_{2}$ & $-a_{5}$ & $a_{2}$ & $-a_{8}$ & $-a_{1}$ \\
\hline$s_{31}^{2} u_{12} u_{23}$ & $a_{5}$ & 0 & $-a_{7}$ & 0 & $a_{2}$ & 0 & 0 & 0 & 0 & 0 & 0 & $-a_{2}$ \\
\hline$s_{31}^{2} u_{12} u_{21}$ & $a_{1}$ & $-a_{7}$ & $a_{8}$ & 0 & $a_{8}$ & $-a_{8}$ & 0 & $a_{8}$ & 0 & 0 & $a_{7}$ & $-a_{1}$ \\
\hline$s_{31}^{3} u_{23}$ & $a_{1}$ & $-a_{7}$ & $-a_{8}$ & $-a_{2}$ & $a_{2}$ & 0 & $-a_{2}$ & 0 & 0 & 0 & $a_{7}$ & 0 \\
\hline$s_{31}^{3} u_{21}$ & $a_{1}$ & $-a_{7}$ & $-a_{8}$ & $-a_{2}$ & $a_{2}$ & 0 & $-a_{2}$ & 0 & 0 & 0 & $a_{7}$ & 0 \\
\hline$s_{23} u_{13} u_{32}^{2}$ & $a_{2}$ & $-a_{7}$ & $-a_{7}$ & $-a_{2}$ & 0 & 0 & $-a_{2}$ & 0 & $-a_{1}$ & $-a_{8}$ & $a_{19}$ & 0 \\
\hline$s_{23} u_{13} u_{31} u_{32}$ & 0 & $-a_{7}$ & $-a_{7}$ & 0 & 0 & 0 & $-a_{1}$ & 0 & $-a_{1}$ & $-a_{2}$ & 0 & $-a_{8}$ \\
\hline$s_{23} u_{13} u_{31}^{2}$ & $a_{2}$ & 0 & 0 & $a_{2}$ & 0 & 0 & 0 & 0 & $-a_{2}$ & 0 & 0 & $-a_{8}$ \\
\hline$s_{23} u_{13} u_{23} u_{32}$ & 0 & $a_{8}$ & $a_{8}$ & 0 & 0 & 0 & $-a_{5}$ & 0 & $a_{2}$ & $a_{8}$ & $-a_{7}$ & $a_{8}$ \\
\hline$s_{23} u_{13} u_{23} u_{31}$ & $a_{1}$ & 0 & 0 & 0 & 0 & 0 & $-a_{1}$ & 0 & $a_{2}$ & 0 & $a_{19}$ & 0 \\
\hline$s_{23} u_{13} u_{23}^{2}$ & $a_{2}$ & $-a_{7}$ & $-a_{7}$ & 0 & 0 & 0 & $a_{2}$ & 0 & 0 & $a_{2}$ & $a_{19}$ & $a_{8}$ \\
\hline$s_{23} u_{13} u_{21} u_{32}$ & $a_{2}$ & $a_{7}$ & $a_{7}$ & 0 & 0 & 0 & $-a_{5}$ & 0 & 0 & $a_{2}$ & $-a_{7}$ & 0 \\
\hline$s_{23} u_{13} u_{21} u_{31}$ & $a_{3}$ & 0 & 0 & $a_{4}$ & 0 & 0 & 0 & 0 & $a_{1}$ & $a_{1}$ & $a_{19}$ & 0 \\
\hline$s_{23} u_{13} u_{21} u_{23}$ & $a_{2}$ & $-a_{8}$ & $-a_{8}$ & $a_{2}$ & 0 & 0 & 0 & 0 & $a_{2}$ & $a_{2}$ & $a_{22}$ & 0 \\
\hline$s_{23} u_{13} u_{21}^{2}$ & $a_{1}$ & $-a_{7}$ & $-a_{7}$ & $a_{2}$ & 0 & 0 & 0 & 0 & $a_{2}$ & $a_{2}$ & $a_{7}$ & 0 \\
\hline$s_{23} u_{13}^{2} u_{32}$ & 0 & $a_{7}$ & $a_{7}$ & $-a_{1}$ & 0 & 0 & 0 & 0 & 0 & $a_{10}$ & 0 & $-a_{10}$ \\
\hline$s_{23} u_{13}^{2} u_{31}$ & 0 & 0 & 0 & $-a_{1}$ & 0 & 0 & $-a_{1}$ & 0 & 0 & $a_{1}$ & 0 & $a_{2}$ \\
\hline$s_{23} u_{13}^{2} u_{23}$ & $a_{1}$ & 0 & 0 & 0 & 0 & 0 & $a_{1}$ & 0 & $-a_{2}$ & $a_{2}$ & $-a_{19}$ & $a_{10}$ \\
\hline$s_{23} u_{13}^{2} u_{21}$ & $-a_{2}$ & 0 & 0 & 0 & 0 & 0 & 0 & 0 & 0 & $a_{2}$ & $-a_{19}$ & $a_{2}$ \\
\hline$s_{23} u_{13}^{3}$ & $a_{2}$ & 0 & 0 & 0 & 0 & 0 & $a_{2}$ & 0 & $-a_{2}$ & $-a_{2}$ & 0 & 0 \\
\hline$s_{23} u_{12} u_{32}^{2}$ & $a_{2}$ & $-a_{7}$ & $-a_{7}$ & 0 & 0 & 0 & $a_{2}$ & 0 & 0 & $a_{8}$ & $a_{19}$ & $a_{2}$ \\
\hline$s_{23} u_{12} u_{31} u_{32}$ & $a_{2}$ & $-a_{8}$ & $-a_{8}$ & $a_{2}$ & 0 & 0 & 0 & 0 & $a_{2}$ & 0 & $a_{22}$ & $a_{2}$ \\
\hline$s_{23} u_{12} u_{31}^{2}$ & $a_{1}$ & $-a_{7}$ & $-a_{7}$ & $a_{2}$ & 0 & 0 & 0 & 0 & $a_{2}$ & 0 & $a_{7}$ & $a_{2}$ \\
\hline$s_{23} u_{12} u_{23} u_{32}$ & 0 & $a_{8}$ & $a_{8}$ & $a_{2}$ & 0 & 0 & $-a_{5}$ & 0 & 0 & $a_{8}$ & $-a_{7}$ & $a_{8}$ \\
\hline$s_{23} u_{12} u_{23} u_{31}$ & $a_{2}$ & $a_{7}$ & $a_{7}$ & 0 & 0 & 0 & $-a_{5}$ & 0 & 0 & 0 & $-a_{7}$ & $a_{2}$ \\
\hline$s_{23} u_{12} u_{23}^{2}$ & $a_{2}$ & $-a_{7}$ & $-a_{7}$ & $-a_{1}$ & 0 & 0 & $-a_{2}$ & 0 & $-a_{2}$ & 0 & $a_{19}$ & $-a_{8}$ \\
\hline$s_{23} u_{12} u_{21} u_{32}$ & $a_{1}$ & 0 & 0 & $a_{2}$ & 0 & 0 & $-a_{1}$ & 0 & 0 & 0 & $a_{19}$ & 0 \\
\hline$s_{23} u_{12} u_{21} u_{31}$ & $a_{3}$ & 0 & 0 & $a_{1}$ & 0 & 0 & 0 & 0 & $a_{4}$ & 0 & $a_{19}$ & $a_{1}$ \\
\hline$s_{23} u_{12} u_{21} u_{23}$ & 0 & $-a_{7}$ & $-a_{7}$ & $-a_{1}$ & 0 & 0 & $-a_{1}$ & 0 & 0 & $-a_{8}$ & 0 & $-a_{2}$ \\
\hline$s_{23} u_{12} u_{21}^{2}$ & $a_{2}$ & 0 & 0 & $-a_{2}$ & 0 & 0 & 0 & 0 & $a_{2}$ & $-a_{8}$ & 0 & 0 \\
\hline$s_{23} u_{12} u_{13} u_{32}$ & $a_{1}$ & $a_{7}$ & $a_{7}$ & $-a_{4}$ & 0 & 0 & 0 & 0 & $-a_{4}$ & $a_{2}$ & $-a_{19}$ & $-a_{8}$ \\
\hline$s_{23} u_{12} u_{13} u_{31}$ & $-a_{2}$ & $a_{7}$ & $a_{7}$ & $-a_{2}$ & 0 & 0 & $-a_{1}$ & 0 & $-a_{2}$ & $a_{1}$ & $-a_{8}$ & $a_{2}$ \\
\hline$s_{23} u_{12} u_{13} u_{23}$ & $a_{1}$ & $a_{7}$ & $a_{7}$ & $-a_{4}$ & 0 & 0 & 0 & 0 & $-a_{4}$ & $-a_{8}$ & $-a_{19}$ & $a_{2}$ \\
\hline$s_{23} u_{12} u_{13} u_{21}$ & $-a_{2}$ & $a_{7}$ & $a_{7}$ & $-a_{2}$ & 0 & 0 & $-a_{1}$ & 0 & $-a_{2}$ & $a_{2}$ & $-a_{8}$ & $a_{1}$ \\
\hline$s_{23} u_{12} u_{13}^{2}$ & $a_{2}$ & 0 & 0 & $-a_{1}$ & 0 & 0 & $a_{2}$ & 0 & $-a_{1}$ & $-a_{10}$ & $a_{7}$ & $-a_{10}$ \\
\hline
\end{tabular}


Table 1. Continued.

\begin{tabular}{|c|c|c|c|c|c|c|c|c|c|c|c|c|}
\hline & $\overline{\bar{A} A}$ & $\overline{\bar{B}}$ & $\overline{c C}$ & 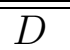 & $\overline{E E}$ & $\overline{F F}$ & $\overline{\bar{G}}$ & $\overline{\bar{H}}$ & $\overline{\overline{I I}}$ & $\overline{\overline{J J}}$ & $\overline{\bar{K}}$ & 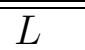 \\
\hline$s_{23} u_{12}^{2} u_{32}$ & $\overline{a_{1}}$ & $\overline{0}$ & 0 & $-a_{2}$ & 0 & 0 & $\overline{a_{1}}$ & 0 & 0 & $a_{10}$ & $-a_{19}$ & $a_{2}$ \\
\hline$s_{23} u_{12}^{2} u_{31}$ & $-a_{2}$ & 0 & 0 & 0 & 0 & 0 & 0 & 0 & 0 & $a_{2}$ & $-a_{19}$ & $a_{2}$ \\
\hline$s_{23} u_{12}^{2} u_{23}$ & 0 & $a_{7}$ & $a_{7}$ & 0 & 0 & 0 & 0 & 0 & $-a_{1}$ & $-a_{10}$ & 0 & $a_{10}$ \\
\hline$s_{23} u_{12}^{2} u_{21}$ & 0 & 0 & 0 & 0 & 0 & 0 & $-a_{1}$ & 0 & $-a_{1}$ & $a_{2}$ & 0 & $a_{1}$ \\
\hline$s_{23} u_{12}^{2} u_{13}$ & $a_{2}$ & 0 & 0 & $-a_{1}$ & 0 & 0 & $a_{2}$ & 0 & $-a_{1}$ & $-a_{10}$ & $a_{7}$ & $-a_{10}$ \\
\hline$s_{23} u_{12}^{3}$ & $a_{2}$ & 0 & 0 & $-a_{2}$ & 0 & 0 & $a_{2}$ & 0 & 0 & 0 & 0 & $-a_{2}$ \\
\hline$s_{23} s_{31} u_{32}^{2}$ & 0 & $a_{8}$ & 0 & $-a_{2}$ & $a_{2}$ & 0 & $-a_{2}$ & 0 & 0 & $-a_{8}$ & 0 & 0 \\
\hline$s_{23} s_{31} u_{31} u_{32}$ & $-a_{1}$ & $a_{7}$ & $a_{9}$ & $-a_{1}$ & $a_{2}$ & $a_{2}$ & 0 & $-a_{2}$ & 0 & 0 & $-a_{22}$ & $-a_{2}$ \\
\hline$s_{23} s_{31} u_{31}^{2}$ & 0 & $-a_{7}$ & $a_{9}$ & $-a_{2}$ & 0 & $a_{2}$ & 0 & 0 & $-a_{2}$ & 0 & $a_{7}$ & $-a_{2}$ \\
\hline$s_{23} s_{31} u_{23} u_{32}$ & $a_{1}$ & $-a_{9}$ & $-a_{7}$ & $a_{1}$ & $-a_{10}$ & $a_{8}$ & 0 & $-a_{8}$ & $-a_{1}$ & $a_{10}$ & $a_{19}$ & $a_{2}$ \\
\hline$s_{23} s_{31} u_{23} u_{31}$ & $a_{5}$ & $-a_{7}$ & $-a_{11}$ & 0 & $-a_{2}$ & 0 & $-a_{5}$ & $a_{2}$ & $-a_{1}$ & 0 & $a_{7}$ & $a_{2}$ \\
\hline$s_{23} s_{31} u_{23}^{2}$ & 0 & $a_{7}$ & $a_{7}$ & $-a_{1}$ & $a_{8}$ & $-a_{8}$ & $-a_{4}$ & $a_{8}$ & $a_{2}$ & 0 & $-a_{19}$ & 0 \\
\hline$s_{23} s_{31} u_{21} u_{32}$ & $a_{2}$ & $-a_{2}$ & $a_{8}$ & 0 & 0 & $a_{2}$ & $a_{1}$ & $-a_{2}$ & 0 & $a_{1}$ & $-a_{7}$ & 0 \\
\hline$s_{23} s_{31} u_{21} u_{31}$ & $a_{3}$ & $-a_{9}$ & 0 & $-a_{2}$ & $-a_{8}$ & $a_{10}$ & 0 & $a_{8}$ & 0 & 0 & $a_{20}$ & 0 \\
\hline$s_{23} s_{31} u_{21} u_{23}$ & $a_{2}$ & $a_{9}$ & $-a_{9}$ & $-a_{2}$ & $-a_{8}$ & $-a_{8}$ & $-a_{6}$ & $a_{10}$ & 0 & $-a_{10}$ & 0 & 0 \\
\hline$s_{23} s_{31} u_{21}^{2}$ & $a_{4}$ & $a_{7}$ & $-a_{8}$ & $-a_{2}$ & $-a_{8}$ & $a_{8}$ & 0 & $a_{8}$ & $a_{2}$ & $-a_{10}$ & $a_{8}$ & 0 \\
\hline$s_{23} s_{31} u_{13} u_{32}$ & $a_{5}$ & $-a_{2}$ & $-a_{8}$ & 0 & 0 & $-a_{2}$ & $-a_{1}$ & $a_{2}$ & $-a_{5}$ & $a_{2}$ & $a_{7}$ & $-a_{1}$ \\
\hline$s_{23} s_{31} u_{13} u_{31}$ & $a_{1}$ & $-a_{8}$ & $-a_{10}$ & $a_{1}$ & $a_{8}$ & $-a_{10}$ & $-a_{1}$ & $-a_{8}$ & 0 & $a_{1}$ & $a_{7}$ & $a_{16}$ \\
\hline$s_{23} s_{31} u_{13} u_{23}$ & $a_{1}$ & $a_{7}$ & 0 & $-a_{6}$ & $a_{2}$ & $a_{2}$ & $-a_{1}$ & 0 & $-a_{1}$ & $a_{15}$ & $-a_{21}$ & $a_{17}$ \\
\hline$s_{23} s_{31} u_{13} u_{21}$ & $a_{2}$ & $-a_{7}$ & $-a_{11}$ & $-a_{1}$ & $a_{2}$ & 0 & $-a_{6}$ & 0 & 0 & $a_{3}$ & $-a_{8}$ & $a_{18}$ \\
\hline$s_{23} s_{31} u_{13}^{2}$ & 0 & $a_{9}$ & $a_{9}$ & $-a_{1}$ & $-a_{8}$ & $a_{8}$ & $a_{2}$ & $a_{8}$ & $-a_{4}$ & $-a_{8}$ & $-a_{8}$ & $-a_{10}$ \\
\hline$s_{23} s_{31} u_{12} u_{32}$ & $a_{3}$ & $-a_{7}$ & $-a_{11}$ & $-a_{2}$ & $a_{10}$ & $-a_{8}$ & 0 & $a_{8}$ & 0 & $a_{2}$ & $a_{21}$ & $a_{4}$ \\
\hline$s_{23} s_{31} u_{12} u_{31}$ & $a_{2}$ & 0 & $-a_{12}$ & 0 & $a_{2}$ & 0 & 0 & $-a_{2}$ & $a_{1}$ & $a_{1}$ & $-a_{7}$ & $a_{6}$ \\
\hline$s_{23} s_{31} u_{12} u_{23}$ & $a_{2}$ & $-a_{7}$ & $a_{8}$ & $-a_{1}$ & 0 & $a_{2}$ & 0 & 0 & $-a_{6}$ & $a_{4}$ & $-a_{20}$ & $a_{15}$ \\
\hline$s_{23} s_{31} u_{12} u_{21}$ & 0 & 0 & 0 & 0 & 0 & 0 & 0 & $-a_{2}$ & 0 & $a_{5}$ & $-a_{9}$ & $a_{18}$ \\
\hline$s_{23} s_{31} u_{12} u_{13}$ & $a_{2}$ & $a_{7}$ & $a_{1}$ & $-a_{2}$ & $-a_{8}$ & $-a_{8}$ & 0 & $a_{10}$ & $-a_{6}$ & $-a_{2}$ & $-a_{21}$ & $-a_{17}$ \\
\hline$s_{23} s_{31} u_{12}^{2}$ & $a_{4}$ & $-a_{7}$ & $a_{8}$ & $-a_{2}$ & $a_{8}$ & $-a_{8}$ & $a_{2}$ & $a_{8}$ & 0 & 0 & $a_{7}$ & $-a_{4}$ \\
\hline$s_{23} s_{31}^{2} u_{32}$ & $a_{1}$ & $a_{7}$ & $-a_{8}$ & $-a_{1}$ & $a_{1}$ & 0 & $-a_{1}$ & 0 & 0 & $-a_{8}$ & $a_{7}$ & 0 \\
\hline$s_{23} s_{31}^{2} u_{31}$ & 0 & 0 & $-a_{9}$ & $-a_{1}$ & $a_{2}$ & $a_{2}$ & 0 & $-a_{2}$ & 0 & $a_{2}$ & $-a_{19}$ & $a_{4}$ \\
\hline$s_{23} s_{31}^{2} u_{23}$ & $a_{1}$ & $-a_{2}$ & $-a_{8}$ & 0 & $-a_{8}$ & $a_{8}$ & 0 & $a_{8}$ & $-a_{5}$ & $a_{15}$ & $-a_{22}$ & $a_{3}$ \\
\hline$s_{23} s_{31}^{2} u_{21}$ & $a_{1}$ & $-a_{8}$ & $-a_{10}$ & $-a_{1}$ & $a_{8}$ & $a_{10}$ & $a_{1}$ & $-a_{10}$ & 0 & $a_{4}$ & $-a_{7}$ & $a_{18}$ \\
\hline$s_{23} s_{31}^{2} u_{13}$ & $a_{1}$ & $-a_{7}$ & $a_{7}$ & $a_{2}$ & $-a_{8}$ & $-a_{8}$ & $-a_{2}$ & $a_{10}$ & $-a_{5}$ & $a_{8}$ & $-a_{22}$ & $-a_{1}$ \\
\hline$s_{23} s_{31}^{2} u_{12}$ & $a_{5}$ & $-a_{8}$ & 0 & $-a_{2}$ & $a_{10}$ & $-a_{8}$ & $-a_{2}$ & $a_{8}$ & 0 & 0 & $a_{8}$ & $-a_{1}$ \\
\hline$s_{23} s_{31}^{3}$ & $a_{1}$ & $-a_{7}$ & $-a_{8}$ & $-a_{2}$ & $a_{2}$ & 0 & $-a_{2}$ & 0 & 0 & 0 & $a_{7}$ & 0 \\
\hline$s_{23}^{2} u_{13} u_{32}$ & 0 & $-a_{7}$ & $a_{8}$ & $-a_{1}$ & $a_{2}$ & 0 & $-a_{5}$ & 0 & 0 & 0 & $-a_{22}$ & 0 \\
\hline$s_{23}^{2} u_{13} u_{31}$ & $a_{1}$ & $-a_{8}$ & $a_{7}$ & 0 & $a_{8}$ & $a_{8}$ & 0 & $-a_{8}$ & 0 & 0 & $a_{8}$ & $-a_{2}$ \\
\hline$s_{23}^{2} u_{13} u_{23}$ & $a_{1}$ & $-a_{7}$ & $-a_{2}$ & $a_{2}$ & $-a_{8}$ & $a_{8}$ & 0 & $a_{8}$ & $-a_{2}$ & $a_{2}$ & $a_{7}$ & $a_{2}$ \\
\hline$s_{23}^{2} u_{13} u_{21}$ & $a_{5}$ & $-a_{9}$ & $-a_{9}$ & 0 & 0 & $a_{2}$ & 0 & 0 & 0 & $a_{2}$ & $a_{9}$ & 0 \\
\hline$s_{23}^{2} u_{13}^{2}$ & 0 & $a_{8}$ & $-a_{7}$ & $a_{1}$ & $-a_{8}$ & $-a_{8}$ & 0 & $a_{8}$ & 0 & $a_{1}$ & $-a_{8}$ & $a_{1}$ \\
\hline
\end{tabular}


Table 1. Continued.

\begin{tabular}{|c|c|c|c|c|c|c|c|c|c|c|c|c|}
\hline & $\overline{\bar{A} A}$ & $\overline{\bar{B}}$ & $\overline{C C}$ & $\overline{c D}$ & $\bar{E}$ & $\overline{F F}$ & $\overline{\bar{G}}$ & $\overline{\bar{H}}$ & $\overline{\overline{I I}}$ & 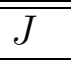 & 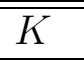 & 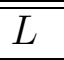 \\
\hline$s_{23}^{2} u_{12} u_{32}$ & $\overline{a_{1}}$ & $-a_{2}$ & $-a_{7}$ & $-a_{2}$ & $a_{8}$ & $\overline{a_{8}}$ & 0 & $-a_{8}$ & $a_{2}$ & $\overline{a_{2}}$ & $a_{7}$ & $a_{2}$ \\
\hline$s_{23}^{2} u_{12} u_{31}$ & $a_{5}$ & $-a_{9}$ & $-a_{9}$ & 0 & 0 & $a_{2}$ & 0 & 0 & 0 & 0 & $a_{9}$ & $a_{2}$ \\
\hline$s_{23}^{2} u_{12} u_{23}$ & 0 & $a_{8}$ & $-a_{7}$ & 0 & 0 & 0 & $-a_{5}$ & $a_{2}$ & $-a_{1}$ & 0 & $-a_{22}$ & 0 \\
\hline$s_{23}^{2} u_{12} u_{21}$ & $a_{1}$ & $a_{7}$ & $-a_{8}$ & 0 & $-a_{8}$ & $a_{8}$ & 0 & $a_{8}$ & 0 & $-a_{2}$ & $a_{8}$ & 0 \\
\hline$s_{23}^{2} u_{12} u_{13}$ & $-a_{1}$ & $a_{2}$ & $a_{2}$ & $a_{2}$ & $a_{8}$ & $-a_{10}$ & $-a_{5}$ & $a_{8}$ & $a_{2}$ & $a_{4}$ & $-a_{23}$ & $a_{4}$ \\
\hline$s_{23}^{2} u_{12}^{2}$ & 0 & $-a_{7}$ & $a_{8}$ & 0 & $a_{8}$ & $-a_{8}$ & 0 & $-a_{8}$ & $a_{1}$ & $a_{1}$ & $-a_{8}$ & $a_{1}$ \\
\hline$s_{23}^{2} s_{31} u_{32}$ & 0 & $-a_{8}$ & $a_{8}$ & $-a_{1}$ & $a_{2}$ & $a_{2}$ & 0 & $-a_{2}$ & 0 & $a_{2}$ & $-a_{7}$ & 0 \\
\hline$s_{23}^{2} s_{31} u_{31}$ & $a_{1}$ & $-a_{9}$ & $a_{7}$ & $-a_{1}$ & 0 & $a_{1}$ & 0 & 0 & $-a_{1}$ & 0 & $a_{9}$ & $-a_{2}$ \\
\hline$s_{23}^{2} s_{31} u_{23}$ & $a_{1}$ & 0 & $-a_{2}$ & $a_{2}$ & $-a_{8}$ & $-a_{8}$ & $-a_{5}$ & $a_{10}$ & $-a_{2}$ & 0 & $a_{19}$ & $a_{2}$ \\
\hline$s_{23}^{2} s_{31} u_{21}$ & $a_{5}$ & $-a_{7}$ & $-a_{2}$ & $-a_{2}$ & $-a_{8}$ & $a_{10}$ & 0 & $a_{8}$ & $-a_{2}$ & $-a_{2}$ & $a_{2}$ & 0 \\
\hline$s_{23}^{2} s_{31} u_{13}$ & $a_{1}$ & 0 & $-a_{11}$ & 0 & $a_{8}$ & $-a_{8}$ & $-a_{5}$ & $a_{8}$ & 0 & $a_{4}$ & $-a_{21}$ & $a_{6}$ \\
\hline$s_{23}^{2} s_{31} u_{12}$ & $a_{1}$ & $-a_{2}$ & $-a_{8}$ & $-a_{1}$ & $a_{10}$ & $a_{8}$ & 0 & $-a_{10}$ & $a_{1}$ & $a_{5}$ & $-a_{8}$ & $a_{6}$ \\
\hline$s_{23}^{2} s_{31}^{2}$ & $a_{1}$ & $-a_{9}$ & $-a_{2}$ & $-a_{1}$ & $a_{2}$ & $a_{2}$ & 0 & $-a_{2}$ & 0 & $a_{1}$ & 0 & $a_{5}$ \\
\hline$s_{23}^{3} u_{13}$ & $a_{1}$ & $-a_{8}$ & $-a_{8}$ & $-a_{2}$ & 0 & $a_{2}$ & 0 & 0 & $-a_{2}$ & 0 & $a_{8}$ & 0 \\
\hline$s_{23}^{3} u_{12}$ & $a_{1}$ & $-a_{8}$ & $-a_{8}$ & $-a_{2}$ & 0 & $a_{2}$ & 0 & 0 & $-a_{2}$ & 0 & $a_{8}$ & 0 \\
\hline$s_{23}^{3} s_{31}$ & $a_{1}$ & $-a_{8}$ & $-a_{8}$ & $-a_{2}$ & 0 & $a_{2}$ & 0 & 0 & $-a_{2}$ & 0 & $a_{8}$ & 0 \\
\hline$s_{12} u_{32}^{3}$ & $a_{2}$ & 0 & 0 & $a_{2}$ & 0 & 0 & $-a_{2}$ & 0 & 0 & $-a_{8}$ & 0 & 0 \\
\hline$s_{12} u_{31} u_{32}^{2}$ & $a_{2}$ & 0 & 0 & $a_{2}$ & 0 & 0 & $-a_{1}$ & 0 & $-a_{1}$ & $-a_{2}$ & 0 & $-a_{2}$ \\
\hline$s_{12} u_{31}^{2} u_{32}$ & $a_{2}$ & 0 & 0 & $a_{2}$ & 0 & 0 & $-a_{1}$ & 0 & $-a_{1}$ & $-a_{2}$ & 0 & $-a_{2}$ \\
\hline$s_{12} u_{31}^{3}$ & $a_{2}$ & 0 & 0 & $a_{2}$ & 0 & 0 & 0 & 0 & $-a_{2}$ & 0 & 0 & $-a_{8}$ \\
\hline$s_{12} u_{23} u_{32}^{2}$ & 0 & 0 & 0 & $-a_{1}$ & 0 & 0 & 0 & 0 & $-a_{1}$ & $a_{2}$ & 0 & $a_{2}$ \\
\hline$s_{12} u_{23} u_{31} u_{32}$ & $-a_{2}$ & 0 & 0 & $-a_{1}$ & 0 & 0 & $-a_{2}$ & 0 & $-a_{2}$ & $a_{2}$ & 0 & $a_{10}$ \\
\hline$s_{12} u_{23} u_{31}^{2}$ & $-a_{2}$ & 0 & 0 & 0 & 0 & 0 & 0 & 0 & 0 & 0 & 0 & $a_{8}$ \\
\hline$s_{12} u_{23}^{2} u_{32}$ & $a_{2}$ & 0 & 0 & 0 & 0 & 0 & $-a_{2}$ & 0 & $a_{2}$ & 0 & 0 & $-a_{8}$ \\
\hline$s_{12} u_{23}^{2} u_{31}$ & $a_{1}$ & 0 & 0 & 0 & 0 & 0 & $a_{2}$ & 0 & $a_{2}$ & 0 & 0 & 0 \\
\hline$s_{12} u_{21} u_{32}^{2}$ & 0 & 0 & 0 & 0 & 0 & 0 & 0 & 0 & $-a_{1}$ & $a_{2}$ & 0 & 0 \\
\hline$s_{12} u_{21} u_{31} u_{32}$ & $a_{1}$ & 0 & 0 & 0 & 0 & 0 & $-a_{4}$ & 0 & $-a_{4}$ & $a_{2}$ & 0 & $a_{8}$ \\
\hline$s_{12} u_{21} u_{31}^{2}$ & $a_{1}$ & 0 & 0 & $a_{1}$ & 0 & 0 & 0 & 0 & $-a_{2}$ & $a_{2}$ & 0 & 0 \\
\hline$s_{12} u_{21} u_{23} u_{32}$ & 0 & 0 & 0 & $-a_{1}$ & 0 & 0 & $-a_{1}$ & 0 & 0 & $-a_{2}$ & 0 & $-a_{8}$ \\
\hline$s_{12} u_{21} u_{23} u_{31}$ & $a_{2}$ & 0 & 0 & 0 & 0 & 0 & $a_{2}$ & 0 & $a_{2}$ & $-a_{2}$ & 0 & 0 \\
\hline$s_{12} u_{21}^{2} u_{32}$ & $a_{2}$ & 0 & 0 & $-a_{2}$ & 0 & 0 & $-a_{1}$ & 0 & $-a_{2}$ & $-a_{8}$ & 0 & 0 \\
\hline$s_{12} u_{21}^{2} u_{31}$ & $a_{2}$ & 0 & 0 & $a_{2}$ & 0 & 0 & 0 & 0 & 0 & 0 & 0 & 0 \\
\hline$s_{12} u_{13} u_{32}^{2}$ & $-a_{2}$ & 0 & 0 & 0 & 0 & 0 & 0 & 0 & 0 & $a_{8}$ & 0 & $-a_{1}$ \\
\hline$s_{12} u_{13} u_{31} u_{32}$ & $-a_{2}$ & $-a_{7}$ & $-a_{7}$ & $-a_{1}$ & 0 & 0 & $-a_{2}$ & 0 & $-a_{2}$ & $a_{14}$ & $a_{7}$ & 0 \\
\hline$s_{12} u_{13} u_{31}^{2}$ & 0 & 0 & 0 & $-a_{1}$ & 0 & 0 & $-a_{1}$ & 0 & 0 & $a_{1}$ & 0 & $a_{2}$ \\
\hline$s_{12} u_{13} u_{23} u_{32}$ & $a_{3}$ & 0 & 0 & 0 & 0 & 0 & $a_{1}$ & 0 & $a_{4}$ & $a_{2}$ & 0 & $a_{1}$ \\
\hline$s_{12} u_{13} u_{23} u_{31}$ & $a_{3}$ & 0 & 0 & 0 & 0 & 0 & $a_{4}$ & 0 & $a_{1}$ & $a_{4}$ & 0 & $a_{2}$ \\
\hline$s_{12} u_{13} u_{21} u_{32}$ & $a_{2}$ & $-a_{7}$ & $-a_{7}$ & $-a_{5}$ & 0 & 0 & 0 & 0 & 0 & $a_{16}$ & $a_{7}$ & $a_{2}$ \\
\hline$s_{12} u_{13} u_{21} u_{31}$ & $a_{1}$ & 0 & 0 & $-a_{1}$ & 0 & 0 & 0 & 0 & $a_{2}$ & $a_{4}$ & 0 & $a_{2}$ \\
\hline
\end{tabular}


Table 1. Continued.

\begin{tabular}{|c|c|c|c|c|c|c|c|c|c|c|c|c|}
\hline & $\overline{\bar{A} A}$ & $\overline{\bar{B}}$ & $\overline{\bar{C} C}$ & 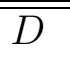 & $\overline{E E}$ & $\overline{F F}$ & $\overline{\bar{G}}$ & $\overline{\bar{H}}$ & 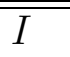 & 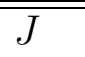 & $\overline{\bar{K}}$ & 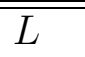 \\
\hline$s_{12} u_{13}^{2} u_{32}$ & $a_{1}$ & $a_{7}$ & $\overline{a_{7}}$ & 0 & 0 & 0 & $\overline{a_{2}}$ & 0 & $a_{2}$ & $-a_{2}$ & $-a_{7}$ & 0 \\
\hline$s_{12} u_{13}^{2} u_{31}$ & $a_{2}$ & 0 & 0 & 0 & 0 & 0 & $a_{2}$ & 0 & $-a_{2}$ & $-a_{2}$ & 0 & 0 \\
\hline$s_{12} u_{12} u_{32}^{2}$ & $a_{1}$ & 0 & 0 & $a_{1}$ & 0 & 0 & $-a_{2}$ & 0 & 0 & $a_{10}$ & 0 & $a_{2}$ \\
\hline$s_{12} u_{12} u_{31} u_{32}$ & $a_{1}$ & $-a_{7}$ & $-a_{7}$ & 0 & 0 & 0 & $-a_{4}$ & 0 & $-a_{4}$ & $a_{15}$ & $a_{7}$ & $a_{1}$ \\
\hline$s_{12} u_{12} u_{31}^{2}$ & 0 & $-a_{7}$ & $-a_{7}$ & 0 & 0 & 0 & $-a_{1}$ & 0 & 0 & $a_{1}$ & $a_{7}$ & $a_{1}$ \\
\hline$s_{12} u_{12} u_{23} u_{32}$ & $a_{1}$ & 0 & 0 & $-a_{1}$ & 0 & 0 & $a_{2}$ & 0 & 0 & $-a_{1}$ & 0 & $-a_{8}$ \\
\hline$s_{12} u_{12} u_{23} u_{31}$ & $a_{2}$ & 0 & 0 & $-a_{5}$ & 0 & 0 & 0 & 0 & 0 & $-a_{1}$ & 0 & $a_{10}$ \\
\hline$s_{12} u_{12} u_{21} u_{32}$ & 0 & $-a_{7}$ & $-a_{7}$ & $-a_{5}$ & 0 & 0 & $a_{2}$ & 0 & 0 & $a_{14}$ & $a_{7}$ & $a_{14}$ \\
\hline$s_{12} u_{12} u_{21} u_{31}$ & 0 & $-a_{7}$ & $-a_{7}$ & $-a_{5}$ & 0 & 0 & 0 & 0 & $a_{2}$ & $a_{10}$ & $a_{7}$ & $a_{4}$ \\
\hline$s_{12} u_{12} u_{13} u_{32}$ & $a_{2}$ & $a_{8}$ & $a_{8}$ & 0 & 0 & 0 & $a_{2}$ & 0 & $a_{2}$ & $-a_{16}$ & $-a_{8}$ & $-a_{5}$ \\
\hline$s_{12} u_{12} u_{13} u_{31}$ & 0 & $a_{7}$ & $a_{7}$ & $-a_{1}$ & 0 & 0 & 0 & 0 & $-a_{1}$ & $-a_{15}$ & $-a_{7}$ & $-a_{4}$ \\
\hline$s_{12} u_{12}^{2} u_{32}$ & $a_{2}$ & $a_{7}$ & $a_{7}$ & $a_{2}$ & 0 & 0 & 0 & 0 & 0 & $-a_{2}$ & $-a_{7}$ & $-a_{2}$ \\
\hline$s_{12} u_{12}^{2} u_{31}$ & $a_{2}$ & $a_{7}$ & $a_{7}$ & $-a_{2}$ & 0 & 0 & $-a_{2}$ & 0 & $-a_{1}$ & $-a_{8}$ & $-a_{7}$ & $-a_{10}$ \\
\hline$s_{12} s_{31} u_{32}^{2}$ & $a_{4}$ & $a_{8}$ & $-a_{8}$ & $a_{2}$ & $a_{8}$ & $a_{8}$ & $-a_{2}$ & $-a_{8}$ & 0 & $-a_{10}$ & $a_{7}$ & 0 \\
\hline$s_{12} s_{31} u_{31} u_{32}$ & $a_{2}$ & $a_{2}$ & $-a_{9}$ & 0 & $-a_{8}$ & $a_{10}$ & $-a_{2}$ & $-a_{8}$ & $-a_{6}$ & $-a_{8}$ & $-a_{7}$ & $a_{1}$ \\
\hline$s_{12} s_{31} u_{31}^{2}$ & 0 & $a_{7}$ & $a_{7}$ & $a_{2}$ & $-a_{8}$ & $a_{8}$ & $-a_{1}$ & $a_{8}$ & $-a_{4}$ & $a_{1}$ & $-a_{7}$ & $a_{2}$ \\
\hline$s_{12} s_{31} u_{23} u_{32}$ & 0 & 0 & 0 & 0 & 0 & $-a_{2}$ & 0 & 0 & 0 & $a_{5}$ & $-a_{8}$ & $a_{5}$ \\
\hline$s_{12} s_{31} u_{23} u_{31}$ & $a_{2}$ & $-a_{2}$ & $-a_{7}$ & $-a_{6}$ & $a_{2}$ & 0 & $-a_{1}$ & 0 & 0 & $a_{6}$ & $-a_{8}$ & $a_{15}$ \\
\hline$s_{12} s_{31} u_{23}^{2}$ & $a_{4}$ & $-a_{8}$ & $a_{8}$ & 0 & $-a_{8}$ & $a_{8}$ & $-a_{2}$ & $a_{8}$ & $a_{2}$ & 0 & $a_{7}$ & $-a_{10}$ \\
\hline$s_{12} s_{31} u_{21} u_{32}$ & $a_{2}$ & $-a_{7}$ & $-a_{2}$ & 0 & 0 & 0 & $-a_{1}$ & $a_{2}$ & $-a_{6}$ & $a_{15}$ & $-a_{8}$ & $a_{6}$ \\
\hline$s_{12} s_{31} u_{21} u_{31}$ & $a_{1}$ & $-a_{7}$ & $-a_{7}$ & $-a_{1}$ & $a_{2}$ & 0 & $-a_{6}$ & $a_{2}$ & $-a_{1}$ & $a_{17}$ & $-a_{8}$ & $a_{17}$ \\
\hline$s_{12} s_{31} u_{21} u_{23}$ & $a_{2}$ & $-a_{9}$ & $a_{2}$ & $-a_{6}$ & $-a_{8}$ & $a_{10}$ & $-a_{2}$ & $-a_{8}$ & 0 & $a_{1}$ & $-a_{7}$ & $-a_{8}$ \\
\hline$s_{12} s_{31} u_{21}^{2}$ & 0 & $a_{7}$ & $a_{7}$ & $-a_{4}$ & $a_{8}$ & $a_{8}$ & $-a_{1}$ & $-a_{8}$ & $a_{2}$ & $a_{2}$ & $-a_{7}$ & $a_{1}$ \\
\hline$s_{12} s_{31} u_{13} u_{32}$ & $a_{2}$ & $-a_{2}$ & $a_{9}$ & 0 & $a_{2}$ & $-a_{2}$ & 0 & 0 & $a_{1}$ & $a_{14}$ & $-a_{9}$ & $-a_{5}$ \\
\hline$s_{12} s_{31} u_{13} u_{31}$ & $a_{1}$ & $-a_{10}$ & $-a_{8}$ & $-a_{1}$ & $a_{8}$ & $-a_{8}$ & $a_{1}$ & $-a_{10}$ & 0 & $a_{14}$ & $a_{8}$ & 0 \\
\hline$s_{12} s_{31} u_{13} u_{23}$ & $a_{3}$ & $a_{7}$ & $-a_{8}$ & 0 & $-a_{8}$ & $a_{8}$ & $-a_{2}$ & $a_{10}$ & 0 & $-a_{1}$ & $a_{8}$ & $a_{1}$ \\
\hline$s_{12} s_{31} u_{13} u_{21}$ & $a_{5}$ & $-a_{10}$ & $-a_{8}$ & $-a_{5}$ & $-a_{2}$ & $a_{2}$ & 0 & 0 & $-a_{1}$ & $a_{1}$ & $a_{8}$ & $a_{2}$ \\
\hline$s_{12} s_{31} u_{13}^{2}$ & 0 & $a_{11}$ & $a_{7}$ & 0 & 0 & 0 & $-a_{2}$ & $a_{2}$ & $-a_{2}$ & $-a_{4}$ & $-a_{7}$ & 0 \\
\hline$s_{12} s_{31} u_{12} u_{32}$ & $a_{3}$ & $-a_{8}$ & $a_{7}$ & 0 & $a_{10}$ & $a_{8}$ & $-a_{2}$ & $-a_{8}$ & 0 & $a_{1}$ & $a_{8}$ & $-a_{1}$ \\
\hline$s_{12} s_{31} u_{12} u_{31}$ & $a_{5}$ & $-a_{8}$ & $-a_{10}$ & $-a_{1}$ & 0 & $a_{2}$ & 0 & $-a_{2}$ & $-a_{5}$ & $a_{2}$ & $a_{8}$ & $a_{1}$ \\
\hline$s_{12} s_{31} u_{12} u_{23}$ & $a_{2}$ & $a_{9}$ & $-a_{2}$ & $a_{1}$ & 0 & $-a_{2}$ & 0 & $a_{2}$ & 0 & $-a_{5}$ & $-a_{9}$ & $a_{14}$ \\
\hline$s_{12} s_{31} u_{12} u_{21}$ & $a_{1}$ & $-a_{8}$ & $-a_{10}$ & 0 & $-a_{10}$ & $-a_{8}$ & $a_{1}$ & $a_{8}$ & $-a_{1}$ & 0 & $a_{8}$ & $a_{14}$ \\
\hline$s_{12} s_{31} u_{12} u_{13}$ & $-a_{1}$ & $a_{10}$ & $a_{10}$ & 0 & $a_{2}$ & $-a_{2}$ & $-a_{1}$ & $a_{2}$ & 0 & $-a_{5}$ & $-a_{10}$ & $-a_{5}$ \\
\hline$s_{12} s_{31} u_{12}^{2}$ & 0 & $a_{7}$ & $a_{11}$ & $-a_{2}$ & $a_{2}$ & 0 & $-a_{2}$ & 0 & 0 & 0 & $-a_{7}$ & $-a_{4}$ \\
\hline$s_{12} s_{31}^{2} u_{32}$ & $a_{5}$ & $a_{7}$ & $-a_{2}$ & $-a_{2}$ & $a_{10}$ & $a_{8}$ & $-a_{2}$ & $-a_{8}$ & 0 & $-a_{2}$ & $a_{8}$ & 0 \\
\hline$s_{12} s_{31}^{2} u_{31}$ & $a_{1}$ & $-a_{7}$ & $-a_{11}$ & $-a_{2}$ & $-a_{8}$ & $a_{10}$ & $a_{2}$ & $-a_{8}$ & $-a_{5}$ & $a_{10}$ & $a_{7}$ & $a_{4}$ \\
\hline$s_{12} s_{31}^{2} u_{23}$ & $a_{1}$ & $-a_{11}$ & $-a_{7}$ & $a_{1}$ & $a_{8}$ & $-a_{10}$ & $-a_{1}$ & $a_{10}$ & 0 & $a_{5}$ & $-a_{7}$ & $a_{4}$ \\
\hline$s_{12} s_{31}^{2} u_{21}$ & $a_{1}$ & $-a_{9}$ & $-a_{10}$ & 0 & $-a_{8}$ & $a_{8}$ & 0 & $a_{8}$ & $-a_{5}$ & $a_{5}$ & 0 & $a_{6}$ \\
\hline$s_{12} s_{31}^{2} u_{13}$ & 0 & 0 & $a_{9}$ & 0 & $a_{2}$ & $-a_{2}$ & $-a_{1}$ & $a_{2}$ & 0 & 0 & $-a_{9}$ & $-a_{1}$ \\
\hline$s_{12} s_{31}^{2} u_{12}$ & $a_{1}$ & 0 & $a_{9}$ & $-a_{1}$ & $a_{1}$ & 0 & $-a_{1}$ & 0 & 0 & 0 & 0 & $-a_{4}$ \\
\hline
\end{tabular}


Table 1. Continued.

\begin{tabular}{|c|c|c|c|c|c|c|c|c|c|c|c|c|}
\hline & $\bar{A}$ & $\bar{B}$ & $\overline{C C}$ & $\overline{c D}$ & $E$ & $\overline{F F}$ & $\overline{\bar{G}}$ & $\overline{\mathrm{H}}$ & $\overline{I I}$ & $\overline{J J}$ & $\bar{~} \bar{K}$ & $\overline{L L}$ \\
\hline$s_{12} s_{31}^{3}$ & $a_{1}$ & $-a_{7}$ & $-a_{8}$ & $-a_{2}$ & $\overline{a_{2}}$ & 0 & $-a_{2}$ & 0 & 0 & 0 & $a_{7}$ & $\overline{0}$ \\
\hline$s_{12} s_{23} u_{32}^{2}$ & 0 & $a_{7}$ & $a_{7}$ & $a_{2}$ & $a_{8}$ & $-a_{8}$ & $-a_{4}$ & $a_{8}$ & $-a_{1}$ & 0 & $-a_{19}$ & 0 \\
\hline$s_{12} s_{23} u_{31} u_{32}$ & $a_{2}$ & $-a_{9}$ & $a_{9}$ & 0 & $a_{10}$ & $-a_{8}$ & $-a_{6}$ & $-a_{8}$ & $-a_{2}$ & 0 & 0 & $-a_{10}$ \\
\hline$s_{12} s_{23} u_{31}^{2}$ & $a_{4}$ & $-a_{8}$ & $a_{7}$ & $a_{2}$ & $a_{8}$ & $a_{8}$ & 0 & $-a_{8}$ & $-a_{2}$ & 0 & $a_{8}$ & $-a_{10}$ \\
\hline$s_{12} s_{23} u_{23} u_{32}$ & $a_{1}$ & $-a_{7}$ & $-a_{9}$ & $-a_{1}$ & $-a_{8}$ & $a_{8}$ & 0 & $-a_{10}$ & $a_{1}$ & $a_{2}$ & $a_{19}$ & $a_{10}$ \\
\hline$s_{12} s_{23} u_{23} u_{31}$ & $a_{2}$ & $a_{8}$ & $-a_{2}$ & 0 & $-a_{2}$ & $a_{2}$ & $a_{1}$ & 0 & 0 & 0 & $-a_{7}$ & $a_{1}$ \\
\hline$s_{12} s_{23} u_{23}^{2}$ & 0 & 0 & $a_{8}$ & 0 & 0 & 0 & $-a_{2}$ & $a_{2}$ & $-a_{2}$ & 0 & 0 & $-a_{8}$ \\
\hline$s_{12} s_{23} u_{21} u_{32}$ & $a_{5}$ & $-a_{11}$ & $-a_{7}$ & $-a_{1}$ & $a_{2}$ & 0 & $-a_{5}$ & $-a_{2}$ & 0 & $a_{2}$ & $a_{7}$ & 0 \\
\hline$s_{12} s_{23} u_{21} u_{31}$ & $a_{3}$ & 0 & $-a_{9}$ & 0 & $a_{8}$ & $a_{10}$ & 0 & $-a_{8}$ & $-a_{2}$ & 0 & $a_{20}$ & 0 \\
\hline$s_{12} s_{23} u_{21} u_{23}$ & $-a_{1}$ & $a_{9}$ & $a_{7}$ & 0 & $-a_{2}$ & $a_{2}$ & 0 & $a_{2}$ & $-a_{1}$ & $-a_{2}$ & $-a_{22}$ & 0 \\
\hline$s_{12} s_{23} u_{21}^{2}$ & 0 & $a_{9}$ & $-a_{7}$ & $-a_{2}$ & 0 & $a_{2}$ & 0 & 0 & $-a_{2}$ & $-a_{2}$ & $a_{7}$ & 0 \\
\hline$s_{12} s_{23} u_{13} u_{32}$ & $a_{2}$ & $a_{8}$ & $-a_{7}$ & $-a_{6}$ & 0 & $a_{2}$ & 0 & 0 & $-a_{1}$ & $a_{15}$ & $-a_{20}$ & $a_{4}$ \\
\hline$s_{12} s_{23} u_{13} u_{31}$ & 0 & 0 & 0 & 0 & $-a_{2}$ & 0 & 0 & 0 & 0 & $a_{18}$ & $-a_{9}$ & $a_{5}$ \\
\hline$s_{12} s_{23} u_{13} u_{23}$ & $a_{3}$ & $-a_{11}$ & $-a_{7}$ & 0 & $a_{8}$ & $-a_{8}$ & 0 & $a_{10}$ & $-a_{2}$ & $a_{4}$ & $a_{21}$ & $a_{2}$ \\
\hline$s_{12} s_{23} u_{13} u_{21}$ & $a_{2}$ & $-a_{12}$ & 0 & $a_{1}$ & $-a_{2}$ & 0 & 0 & $a_{2}$ & 0 & $a_{6}$ & $-a_{7}$ & $a_{1}$ \\
\hline$s_{12} s_{23} u_{13}^{2}$ & $a_{4}$ & $a_{8}$ & $-a_{7}$ & 0 & $a_{8}$ & $-a_{8}$ & $a_{2}$ & $a_{8}$ & $-a_{2}$ & $-a_{4}$ & $a_{7}$ & 0 \\
\hline$s_{12} s_{23} u_{12} u_{32}$ & $a_{1}$ & 0 & $a_{7}$ & $-a_{1}$ & 0 & $a_{2}$ & $-a_{1}$ & $a_{2}$ & $-a_{6}$ & $a_{17}$ & $-a_{21}$ & $a_{15}$ \\
\hline$s_{12} s_{23} u_{12} u_{31}$ & $a_{2}$ & $-a_{11}$ & $-a_{7}$ & 0 & 0 & 0 & $-a_{6}$ & $a_{2}$ & $-a_{1}$ & $a_{18}$ & $-a_{8}$ & $a_{3}$ \\
\hline$s_{12} s_{23} u_{12} u_{23}$ & $a_{5}$ & $-a_{8}$ & $-a_{2}$ & $-a_{5}$ & $a_{2}$ & $-a_{2}$ & $-a_{1}$ & 0 & 0 & $-a_{1}$ & $a_{7}$ & $a_{2}$ \\
\hline$s_{12} s_{23} u_{12} u_{21}$ & $a_{1}$ & $-a_{10}$ & $-a_{8}$ & 0 & $-a_{8}$ & $-a_{10}$ & $-a_{1}$ & $a_{8}$ & $a_{1}$ & $a_{16}$ & $a_{7}$ & $a_{1}$ \\
\hline$s_{12} s_{23} u_{12} u_{13}$ & $a_{2}$ & $a_{1}$ & $a_{7}$ & $-a_{6}$ & $a_{10}$ & $-a_{8}$ & 0 & $-a_{8}$ & $-a_{2}$ & $-a_{17}$ & $-a_{21}$ & $-a_{2}$ \\
\hline$s_{12} s_{23} u_{12}^{2}$ & 0 & $a_{9}$ & $a_{9}$ & $-a_{4}$ & $a_{8}$ & $a_{8}$ & $a_{2}$ & $-a_{8}$ & $-a_{1}$ & $-a_{10}$ & $-a_{8}$ & $-a_{8}$ \\
\hline$s_{12} s_{23} s_{31} u_{32}$ & $a_{5}$ & $-a_{7}$ & $-a_{10}$ & 0 & $a_{2}$ & 0 & $-a_{5}$ & $a_{2}$ & $-a_{6}$ & $a_{16}$ & $-a_{7}$ & $a_{6}$ \\
\hline$s_{12} s_{23} s_{31} u_{31}$ & $a_{5}$ & $-a_{12}$ & $-a_{11}$ & 0 & 0 & $a_{2}$ & $-a_{6}$ & $a_{2}$ & $-a_{5}$ & $a_{18}$ & 0 & $a_{17}$ \\
\hline$s_{12} s_{23} s_{31} u_{23}$ & $a_{5}$ & $-a_{10}$ & $-a_{7}$ & $-a_{6}$ & $a_{2}$ & 0 & $-a_{5}$ & $a_{2}$ & 0 & $a_{6}$ & $-a_{7}$ & $a_{16}$ \\
\hline$s_{12} s_{23} s_{31} u_{21}$ & $a_{5}$ & $-a_{11}$ & $-a_{12}$ & $-a_{5}$ & $a_{2}$ & $a_{2}$ & $-a_{6}$ & 0 & 0 & $a_{17}$ & 0 & $a_{18}$ \\
\hline$s_{12} s_{23} s_{31} u_{13}$ & $a_{5}$ & $a_{7}$ & $-a_{8}$ & $-a_{6}$ & 0 & $a_{2}$ & 0 & $a_{2}$ & $-a_{5}$ & $a_{14}$ & $-a_{21}$ & $a_{4}$ \\
\hline$s_{12} s_{23} s_{31} u_{12}$ & $a_{5}$ & $-a_{8}$ & $a_{7}$ & $-a_{5}$ & $a_{2}$ & $a_{2}$ & 0 & 0 & $-a_{6}$ & $a_{4}$ & $-a_{21}$ & $a_{14}$ \\
\hline$s_{12} s_{23} s_{31}^{2}$ & $a_{6}$ & $-a_{12}$ & $-a_{13}$ & $-a_{2}$ & $a_{8}$ & $a_{8}$ & $-a_{2}$ & $a_{8}$ & $-a_{5}$ & $a_{15}$ & $a_{22}$ & $a_{6}$ \\
\hline$s_{12} s_{23}^{2} u_{32}$ & $a_{1}$ & $-a_{2}$ & 0 & $-a_{2}$ & $a_{10}$ & $-a_{8}$ & $-a_{5}$ & $-a_{8}$ & $a_{2}$ & $a_{2}$ & $a_{19}$ & 0 \\
\hline$s_{12} s_{23}^{2} u_{31}$ & $a_{5}$ & $-a_{2}$ & $-a_{7}$ & $-a_{2}$ & $a_{8}$ & $a_{10}$ & 0 & $-a_{8}$ & $-a_{2}$ & 0 & $a_{2}$ & $-a_{2}$ \\
\hline$s_{12} s_{23}^{2} u_{23}$ & 0 & $a_{8}$ & $-a_{8}$ & 0 & $-a_{2}$ & $a_{2}$ & 0 & $a_{2}$ & $-a_{1}$ & 0 & $-a_{7}$ & $a_{2}$ \\
\hline$s_{12} s_{23}^{2} u_{21}$ & $a_{1}$ & $a_{7}$ & $-a_{9}$ & $-a_{1}$ & 0 & $a_{1}$ & 0 & 0 & $-a_{1}$ & $-a_{2}$ & $a_{9}$ & 0 \\
\hline$s_{12} s_{23}^{2} u_{13}$ & $a_{1}$ & $-a_{8}$ & $-a_{2}$ & $a_{1}$ & $-a_{10}$ & $a_{8}$ & 0 & $a_{10}$ & $-a_{1}$ & $a_{6}$ & $-a_{8}$ & $a_{5}$ \\
\hline$s_{12} s_{23}^{2} u_{12}$ & $a_{1}$ & $-a_{11}$ & 0 & 0 & $a_{8}$ & $-a_{8}$ & $-a_{5}$ & $a_{8}$ & 0 & $a_{6}$ & $-a_{21}$ & $a_{4}$ \\
\hline$s_{12} s_{23}^{2} s_{31}$ & $a_{6}$ & $-a_{13}$ & $-a_{13}$ & $-a_{2}$ & $a_{8}$ & $a_{8}$ & $-a_{5}$ & $a_{8}$ & $-a_{2}$ & $a_{6}$ & $a_{22}$ & $a_{6}$ \\
\hline$s_{12} s_{23}^{3}$ & $a_{1}$ & $-a_{8}$ & $-a_{8}$ & $-a_{2}$ & 0 & $a_{2}$ & 0 & 0 & $-a_{2}$ & 0 & $a_{8}$ & 0 \\
\hline$s_{12}^{2} u_{32}^{2}$ & 0 & $a_{8}$ & $-a_{8}$ & 0 & $a_{8}$ & $-a_{8}$ & 0 & $-a_{8}$ & $a_{1}$ & $a_{2}$ & $-a_{7}$ & $a_{1}$ \\
\hline$s_{12}^{2} u_{31} u_{32}$ & $-a_{1}$ & $a_{7}$ & 0 & $-a_{5}$ & $a_{8}$ & $a_{8}$ & $a_{2}$ & $-a_{10}$ & $a_{2}$ & $a_{3}$ & $-a_{9}$ & $a_{5}$ \\
\hline$s_{12}^{2} u_{31}^{2}$ & 0 & $-a_{8}$ & $a_{7}$ & 0 & $-a_{8}$ & $a_{8}$ & $a_{1}$ & $-a_{8}$ & 0 & $a_{5}$ & $-a_{7}$ & $a_{2}$ \\
\hline$s_{12}^{2} u_{23} u_{32}$ & $a_{1}$ & $-a_{8}$ & $a_{8}$ & 0 & $-a_{8}$ & $a_{8}$ & 0 & $a_{8}$ & 0 & 0 & $a_{7}$ & $-a_{2}$ \\
\hline$s_{12}^{2} u_{23} u_{31}$ & $a_{5}$ & $-a_{8}$ & $-a_{7}$ & 0 & 0 & 0 & 0 & $a_{2}$ & 0 & 0 & $a_{7}$ & 0 \\
\hline
\end{tabular}


Table 1. Continued.

\begin{tabular}{|c|c|c|c|c|c|c|c|c|c|c|c|c|}
\hline & $\bar{A}$ & $\bar{B}$ & $\bar{C}$ & $\bar{D}$ & $\bar{E}$ & $\bar{F}$ & $\bar{G}$ & $\bar{H}$ & $\overline{I I}$ & $\overline{J J}$ & $\bar{K}$ & $\bar{L}$ \\
\hline$s_{12}^{2} u_{21} u_{32}$ & 0 & $-a_{9}$ & $a_{7}$ & $-a_{5}$ & 0 & $\overline{a_{2}}$ & 0 & 0 & $-a_{1}$ & $a_{4}$ & $-a_{7}$ & $a_{10}$ \\
\hline$s_{12}^{2} u_{21} u_{31}$ & $a_{1}$ & $-a_{2}$ & 0 & 0 & $-a_{8}$ & $a_{8}$ & $a_{2}$ & $a_{8}$ & $-a_{2}$ & $a_{4}$ & 0 & $a_{8}$ \\
\hline$s_{12}^{2} u_{13} u_{32}$ & $a_{5}$ & $-a_{7}$ & 0 & 0 & 0 & 0 & 0 & $a_{2}$ & 0 & $-a_{2}$ & 0 & 0 \\
\hline$s_{12}^{2} u_{13} u_{31}$ & $a_{1}$ & $a_{8}$ & $-a_{7}$ & 0 & $a_{8}$ & $-a_{8}$ & 0 & $a_{8}$ & 0 & $-a_{1}$ & $a_{7}$ & 0 \\
\hline$s_{12}^{2} u_{12} u_{32}$ & $a_{1}$ & $a_{7}$ & $-a_{8}$ & 0 & $a_{8}$ & $-a_{8}$ & $-a_{2}$ & $a_{8}$ & $a_{2}$ & $-a_{1}$ & $-a_{7}$ & 0 \\
\hline$s_{12}^{2} u_{12} u_{31}$ & 0 & $a_{8}$ & $-a_{7}$ & $-a_{5}$ & $a_{2}$ & 0 & $-a_{1}$ & 0 & 0 & $-a_{1}$ & $-a_{8}$ & $a_{2}$ \\
\hline$s_{12}^{2} s_{31} u_{32}$ & $a_{1}$ & $-a_{7}$ & $-a_{11}$ & 0 & $a_{10}$ & $-a_{10}$ & $-a_{1}$ & $a_{8}$ & $a_{1}$ & $a_{4}$ & $-a_{7}$ & $a_{5}$ \\
\hline$s_{12}^{2} s_{31} u_{31}$ & $a_{1}$ & $-a_{10}$ & $-a_{9}$ & $-a_{5}$ & $a_{8}$ & $a_{8}$ & 0 & $-a_{8}$ & 0 & $a_{6}$ & 0 & $a_{5}$ \\
\hline$s_{12}^{2} s_{31} u_{23}$ & $a_{5}$ & $-a_{2}$ & $a_{7}$ & 0 & $-a_{8}$ & $a_{8}$ & $-a_{2}$ & $a_{10}$ & $-a_{2}$ & 0 & $a_{8}$ & $-a_{2}$ \\
\hline$s_{12}^{2} s_{31} u_{21}$ & $a_{1}$ & $-a_{11}$ & $-a_{7}$ & $-a_{5}$ & $-a_{8}$ & $a_{10}$ & $a_{2}$ & $-a_{8}$ & $-a_{2}$ & $a_{4}$ & $a_{7}$ & $a_{10}$ \\
\hline$s_{12}^{2} s_{31} u_{13}$ & $a_{1}$ & $a_{9}$ & 0 & 0 & 0 & 0 & $-a_{1}$ & $a_{1}$ & $-a_{1}$ & $-a_{4}$ & 0 & 0 \\
\hline$s_{12}^{2} s_{31} u_{12}$ & 0 & $a_{9}$ & 0 & 0 & $a_{2}$ & $-a_{2}$ & $-a_{1}$ & $a_{2}$ & 0 & $-a_{1}$ & $-a_{9}$ & 0 \\
\hline$s_{12}^{2} s_{31}^{2}$ & $a_{1}$ & $-a_{9}$ & $-a_{9}$ & 0 & $a_{2}$ & $-a_{2}$ & $-a_{1}$ & $a_{2}$ & 0 & $a_{1}$ & 0 & $a_{1}$ \\
\hline$s_{12}^{2} s_{23} u_{32}$ & $a_{1}$ & $-a_{8}$ & $-a_{2}$ & $-a_{5}$ & $a_{8}$ & $a_{8}$ & 0 & $-a_{8}$ & 0 & $a_{3}$ & $-a_{22}$ & $a_{15}$ \\
\hline$s_{12}^{2} s_{23} u_{31}$ & $a_{1}$ & $-a_{10}$ & $-a_{8}$ & 0 & $-a_{10}$ & $a_{10}$ & $a_{1}$ & $a_{8}$ & $-a_{1}$ & $a_{18}$ & $-a_{7}$ & $a_{4}$ \\
\hline$s_{12}^{2} s_{23} u_{23}$ & $a_{1}$ & $-a_{8}$ & $a_{7}$ & 0 & 0 & 0 & $-a_{1}$ & $a_{1}$ & $-a_{1}$ & 0 & $a_{7}$ & $-a_{8}$ \\
\hline$s_{12}^{2} s_{23} u_{21}$ & 0 & $-a_{9}$ & 0 & 0 & $-a_{2}$ & $a_{2}$ & 0 & $a_{2}$ & $-a_{1}$ & $a_{4}$ & $-a_{19}$ & $a_{2}$ \\
\hline$s_{12}^{2} s_{23} u_{13}$ & $a_{5}$ & 0 & $-a_{8}$ & 0 & $a_{8}$ & $-a_{8}$ & $-a_{2}$ & $a_{10}$ & $-a_{2}$ & $-a_{1}$ & $a_{8}$ & 0 \\
\hline$s_{12}^{2} s_{23} u_{12}$ & $a_{1}$ & $a_{7}$ & $-a_{7}$ & $-a_{5}$ & $a_{10}$ & $-a_{8}$ & $-a_{2}$ & $-a_{8}$ & $a_{2}$ & $-a_{1}$ & $-a_{22}$ & $a_{8}$ \\
\hline$s_{12}^{2} s_{23} s_{31}$ & $a_{6}$ & $-a_{13}$ & $-a_{12}$ & $-a_{5}$ & $a_{8}$ & $a_{8}$ & $-a_{2}$ & $a_{8}$ & $-a_{2}$ & $a_{6}$ & $a_{22}$ & $a_{15}$ \\
\hline$s_{12}^{2} s_{23}^{2}$ & $a_{1}$ & $-a_{2}$ & $-a_{9}$ & 0 & $-a_{2}$ & $a_{2}$ & 0 & $a_{2}$ & $-a_{1}$ & $a_{5}$ & 0 & $a_{1}$ \\
\hline$s_{12}^{3} u_{32}$ & $a_{1}$ & $-a_{8}$ & $-a_{7}$ & 0 & 0 & 0 & $-a_{2}$ & $a_{2}$ & $-a_{2}$ & 0 & $a_{7}$ & 0 \\
\hline$s_{12}^{3} u_{31}$ & $a_{1}$ & $-a_{8}$ & $-a_{7}$ & 0 & 0 & 0 & $-a_{2}$ & $a_{2}$ & $-a_{2}$ & 0 & $a_{7}$ & 0 \\
\hline$s_{12}^{3} s_{31}$ & $a_{1}$ & $-a_{8}$ & $-a_{7}$ & 0 & 0 & 0 & $-a_{2}$ & $a_{2}$ & $-a_{2}$ & 0 & $a_{7}$ & 0 \\
\hline$s_{12}^{3} s_{23}$ & $a_{1}$ & $-a_{8}$ & $-a_{7}$ & 0 & 0 & 0 & $-a_{2}$ & $a_{2}$ & $-a_{2}$ & 0 & $a_{7}$ & 0 \\
\hline
\end{tabular}

